\title{
Anomalías y mecanismos de transporte de mercurio en un sistema hidrotermal asociado a fallas normales en Araró, Michoacán (occidente de México)
}

\author{
Isabel Pérez-Martínez ${ }^{1, *}$, Ruth Esther Villanueva-Estrada ${ }^{2}$, \\ Rocío García-Martínez ${ }^{3}$, Augusto Rodríguez-Díaz ${ }^{4}$ y Carles Canet ${ }^{3}$
}

${ }^{1}$ Cátedra CONACyT, Instituto de Geofísica, Universidad Nacional Autónoma de México, Circuito de la Investigación Científica s/n, Ciudad Universitaria, Delegación Coyoacán, 04510, Ciudad de México, México.

${ }^{2}$ Instituto de Geofísica, Unidad Michoacán, Universidad Nacional Autónoma de México, Km 8 Antigua carretera a Pátzcuaro 8701, Col. Ex Hacienda de San José de la Huerta, Morelia, Mich., C.P. 58190, México.

${ }^{3}$ Centro de Ciencias de la Atmósfera, Universidad Nacional Autónoma de México, Circuito de la Investigación Científica s/n, Ciudad Universitaria, Delegación Coyoacán, 04510, Ciudad de México, México.

${ }^{4}$ Instituto de Geofísica, Universidad Nacional Autónoma de México, Circuito de la Investigación Científica s/n, Ciudad Universitaria, Delegación Coyoacán, 04510, Ciudad de México, México.

*perezm@igeofisica.unam.mx

\section{RESUMEN}

En este trabajo se infieren los mecanismos de transporte y movilidad del mercurio en el sistema geotérmico Araró-Simirao, que se asocian a un sistema de fallas de tipo normal y orientación general NE-SW, a través de la modelación hidrogeoquímica del Hg en agua y suelos y su relación con la concentración de $\mathrm{Hg}$ en el aire. Se realizó un muestreo de agua termal y suelos, y se comparó con las emanaciones de gases de mercurio elemental. Las especies predominantes de $\mathrm{Hg}$ en agua son $\mathrm{Hg}^{0}{ }_{\text {(ac) }}, \mathrm{HgCl}_{2}$ y $\mathrm{HgCl}_{3}$, aunque la abundancia de $\mathrm{Hg}^{0}{ }_{\text {(ac) }}$ es menor con respecto a $\mathrm{Hg}^{2+}$. En suelo se encontraron minerales de alteración hidrotermal como barita y partículas con contenidos de Hg. De forma general, se deduce que los procesos geoquímicos que se llevan a cabo para el transporte de $\mathrm{Hg}$ entre los compartimentos ambientales agua, suelo y atmósfera son: volatilización, oxidación, complejación y depósito. El sistema hidrotermal a profundidad favorece el transporte de mercurio elemental en estado gaseoso, de probable origen magmático, hacia la superficie por las zonas de mayor permeabilidad, como es la falla Araró-Simirao; en su ascenso interacciona con el acuífero subsuperficial, enriqueciéndolo en $\mathrm{Hg}$ y formando complejos clorurados que son transportados en el medio acuoso. Al descargarse a la superficie el agua termal enriquecida en $\mathrm{Hg}$, éste se volatiliza como $\mathrm{Hg}_{(\mathrm{g})}$ y permanece en la atmósfera o puede depositarse en los suelos aledaños a los manantiales, adhiriéndose a partículas de materia orgánica o arcillas y formando aureolas de anomalías de $\mathrm{Hg}$ en superficie. De acuerdo con la distribución espacial de estas aureolas, la zona más cercana a los fluidos geotérmicos (ascenso vertical de los gases) es la parte sur del sistema.

Palabras clave: transporte de mercurio; sistema hidrotermal; modelación hidrogeoquímica; oxidación; complejación; volatilización; Araró; Michoacán; México.

\begin{abstract}
In this paper, we infer a mechanism for Hg transport and mobility in the Araró-Simirao geothermal system, which is associated with a normal fault system of general NE-SW orientation, through the hydrogeochemical modeling of $\mathrm{Hg}$ in water and soils and their relationship with the Hg concentration in the air. A sampling of thermal water and soils was carried out, and it was compared with gaseous elemental $\mathrm{Hg}$ emissions. The predominant $\mathrm{Hg}$ species in water are $\mathrm{Hg}_{(a q)}^{0}, \mathrm{HgCl}_{2}$ and $\mathrm{HgCl}_{3}$, although the $\mathrm{Hg}_{(\text {aq) }}^{0}$ abundance is lower with respect to $\mathrm{Hg}^{2+}$. Hydrothermal alteration minerals such as barite and particles with $\mathrm{Hg}$ contents were found in the soil. In general, are inferred the geochemical processes involved in the $\mathrm{Hg}$ transport between the environmental compartments, water, soil, and atmosphere are volatilization, oxidation, complexation, and deposition. The deep hydrothermal system favors the transport of elemental gaseous mercury, of probable magmatic origin, to the surface through the areas with the highest permeability, such as the Araró-Simirao fault. In its ascent, the hydrothermal fluids interact with the subsurface aquifer, enriching the aquifer in $\mathrm{Hg}$ and forming chlorinated complexes that are transported in the aqueous medium. When thermal water enriched in $\mathrm{Hg}$ is discharged to the surface, the mercury volatilizes as $\mathrm{Hg}_{(\mathrm{g})}$ and remains in the atmosphere or can be deposited on the soils surrounding the springs, adhering to particles of organic matter or clays, and forming aureoles of $\mathrm{Hg}$ anomalies on the surface. According to the spatial distribution of the anomalies, the area closest to geothermal fluids (vertical rise of gases) is the southern part of the system.
\end{abstract}

Key words: mercury transport; hydrothermal system; hydrogeochemical modeling; oxidation; complexation; volatilization; Araró; Michoacán; Mexico. 


\section{INTRODUCCIÓN}

El mercurio es un metal de transición, tóxico y volátil que proviene de fuentes antropogénicas y naturales (Varekamp y Buseck, 1986; Rasmussen et al., 1998; Gustin, 2003; Bagnato et al., 2018; Steenhuisen y Wilson, 2019; Sun et al., 2020). Es movilizado naturalmente de los reservorios profundos en la corteza a la atmósfera a través de la actividad volcánica y geológica por lo que, de forma global, el mercurio se encuentra enriquecido en zonas asociadas a los límites tectónicos (Gustin et al., 1999; Selin, 2009).El contenido promedio de Hg en la corteza superior es de 0.5 ppm (Hazen et al. 2012; Beckers y Rinklebe 2017), mientras que la mayoría de las rocas ígneas presentan un contenido menor a 0.2 ppm (Adriano, 2001; Beckers y Rinklebe, 2017). Por otro lado, en suelos no contaminados se han reportado concentraciones de Hg desde 0.01 hasta 1.8 ppm (Han, 2007; Kabata-Pendias, 2011) y en aguas naturales varía de acuerdo con el tipo de cuerpo de agua (ríos: $1 \times 10^{-6}-5 \times 10^{-6} \mathrm{ppm}$; lagos: $0.2 \times 10^{-6}-8 \times 10^{-5} \mathrm{ppm}$, aguas subterráneas: $0.1 \times 10^{-6}-1.6 \times 10^{-5} \mathrm{ppm}$, lluvia: $5 \times 10^{-6}-9 \times 10^{-5} \mathrm{ppm}$; Leopold et al., 2010; Beckers y Rinklebe, 2017). En la atmósfera se ha reportado una concentración media de $1.6 \times 10^{-9} \mathrm{~g} / \mathrm{m}^{3}$ (USEPA, 1997; Selin, 2009). Estas concentraciones están relacionadas con el ciclo biogeoquímico del mercurio, el cual involucra los procesos de transporte atmosférico, depósito en suelos y océanos y revolatilización (Selin, 2009). El tiempo que permanece el mercurio en zonas enriquecidas en este metal, proveniente de fuentes naturales y antropogénicas, es de $10^{3} \mathrm{a} \sim 10^{9}$ años; mientras que en zonas puntuales de emisión de $\mathrm{Hg}$ antropogénico es de aproximadamente 50 años (Gustin et al., 1999).

El mercurio existe en tres estados de oxidación: $\mathrm{Hg}^{0}$ (elemental), $\mathrm{Hg}_{2}{ }^{2+}$ (ion mercuroso) y $\mathrm{Hg}^{2+}$ (ion mercúrico). En la atmósfera, el mercurio se encuentra principalmente en forma elemental en estado gaseoso (GEM: gaseous elemental mercury o $\mathrm{Hg}^{0}$ ), el cual representa aproximadamente el 98\% del Hg atmosférico (Lindqvist et al., 1991; Schroeder y Munthe, 1998; Bagnato et al., 2007; Cabassi et al., 2017). El GEM es la especie química predominante en el transporte del $\mathrm{Hg}$, con un tiempo de residencia en la atmósfera de aproximadamente un año (Gustin et al., 1997). Además, también se encuentra en estado oxidado gaseoso como $\mathrm{Hg}^{2+}$ (GOM: gaseous oxidized mercury, y/o como mercurio particulado (PBM: particulate-bound mercury, $\mathrm{HgP}$ ) (Yu et al., 2018), los cuales precipitan rápidamente vía depósito húmedo o seco. En solución acuosa, el mercurio existe en diversas especies químicas (p. ej., mercurio elemental $\left(\mathrm{Hg}^{0}, \mathrm{HgS}, \mathrm{HgCl}_{2}, \mathrm{CH}_{3} \mathrm{Hg}^{+}\right)$, pero también en especies químicas volátiles (p. ej., $\mathrm{Hg}^{0},\left(\mathrm{CH}_{3}\right)_{2} \mathrm{Hg}, \mathrm{CH}_{3} \mathrm{HgCl}$ ) (Gustin et al., 1999). Bajo condiciones oxidantes el $\mathrm{Hg}^{0}$ (ac) es la especie más importante en solución acuosa (Fein y Williams-Jones, 1997), por lo que en condiciones relativamente oxidantes y alcalinas es posible el transporte significativo del $\mathrm{Hg}_{\text {(ac) }}$. Por otra parte, la presencia y estabilidad de las especies químicas de mercurio en el suelo dependen también de las condiciones óxido-reductoras, por lo que en soluciones acuosas presentes en el suelo solo se produce una diminuta fracción de $\mathrm{Hg}^{2+}$, la cual está unida a los minerales del suelo o adsorbida en superficies inorgánicas u orgánicas sólidas, formado en ocasiones el compuesto metil-mercurio que es una de las especies orgánicas más tóxicas (Fein y Williams-Jones, 1997; Engle et al., 2006).

En sistemas hidrotermales, el mercurio y otros metales base se transportan y se depositan formando complejos iónicos con ligantes presentes en el fluido. En estas soluciones, los ligantes más importantes que forman complejos metálicos son $\mathrm{Cl}^{-}, \mathrm{NH}_{3}$, $\mathrm{HS}^{-}$y $\mathrm{S}^{2-}$ (Barnes et al., 1967). En sistemas hidrotermales asociados a sulfuros, el desarrollo de fases orgánicas controla la movilidad del Hg (Fein y Williams-Jones, 1997). Cuando el fluido hidrotermal es rico en sulfuros a temperaturas mayores a $200^{\circ} \mathrm{C}$, los ligantes de azufre juegan un papel importante para el transporte de mercurio a la superficie (Barnes et al., 1967).
Sin embargo, en aguas con alta salinidad, la formación de complejos clorurados de mercurio es la que juega un rol predominante en el transporte (Varekamps y Buseck, 1984). Experimentos realizados por Jiménez-Moreno et al. (2013) demuestran que el $\mathrm{Hg}^{2+}$ tiende a formar complejos químicos clorurados $\left(\mathrm{HgCl}^{+}, \mathrm{HgCl}_{2}, \mathrm{HgCl}_{3}{ }^{-}\right.$y $\left.\mathrm{HgCl}_{4}{ }^{2-}\right)$ cuando la concentración de cloruros es del orden de $0.5 \mathrm{M} \mathrm{(3.5 \%} \mathrm{de}$ salinidad). El compuesto sólido más abundante de mercurio es el $\mathrm{HgS}$, el cual ocurre en la naturaleza en tres polimorfos: cinabrio, metacinabrio e hipercinabrio (Hazen et al., 2012; Beckers and Rinklebe, 2017); los dos últimos compuestos son los polimorfos de alta temperatura del cinabrio (Hazen et al., 2012). El depósito de minerales sulfurados de mercurio es dependiente del $\mathrm{pH}$ y de las condiciones óxido-reductoras del ambiente o cuando ocurre un decremento en la temperatura (Varekamps y Buseck, 1984).

Como se mencionó anteriormente, en ambientes geotérmicos el transporte de fluidos puede facilitar la lixiviación, la movilidad, el depósito y la concentración de mercurio, dado que fuentes de calor del subsuelo facilitan la volatilización de $\mathrm{Hg}_{(\mathrm{g})}{ }_{\mathrm{g}}$ y su movimiento hacia la superficie (Gustin, 2003). Las estructuras geológicas como fallas, fracturas y contactos litológicos funcionan a menudo como zonas de transporte de esos fluidos y como zonas de precipitación mineral (Sun et al., 2020), por lo que el Hg puede usarse para delinear áreas de actividad hidrotermal fósil (Henneberger, 1986; Nicholson, 1993). Sin embargo, son pocos o nulos los trabajos relativos al transporte de $\mathrm{Hg}$ en sistemas hidrotermales en México, dado que los estudios reportados tratan solo temas de la precipitación de minerales y contaminación de Hg en sedimentos, suelos y plantas por emanaciones de $\mathrm{Hg}$ de ventilas hidrotermales y campos geotérmicos (Prol-Ledesma et al., 2002; Canet et al., 2005; Leal-Acosta 2010, 2013; Pastrana-Corral et al., 2016). Además, son escasos los estudios sobre las emisiones de Hg en zonas de subducción continental o en otros contextos tectónicos (Varekamps y Buseck, 1986; Gustin, 2003; Engle et al., 2006; Sun et al., 2020). Por otro lado, es importante evaluar la liberación de mercurio al ambiente, así como su presencia y comportamiento en los acuíferos cercanos a zonas geotérmicas. Las aguas subterráneas pueden contener apreciables cantidades de mercurio como resultado de la lixiviación de rocas ricas en materia orgánica (Beckers y Rinklebe, 2017) y, por tanto, pueden ser una fuente de emisiones de mercurio elemental hacia la atmósfera.

En este trabajo se reportan los resultados de un estudio orientado a inferir los mecanismos de transporte y movilidad del $\mathrm{Hg}$, derivados de la actividad hidrotermal en el sistema geotérmico Araró-Simirao, a través de la modelación hidrogeoquímica del Hg en agua y suelos y su relación con la concentración de $\mathrm{Hg}$ en el aire.

\section{ÁREA DE ESTUDIO}

El área de estudio se ubica en el estado de Michoacán, en el extremo este de la cuenca del Lago de Cuitzeo (Figura 1a) y forma parte del campo volcánico Michoacán-Guanajuato (Pola et al., 2016). El sistema lacustre de Cuitzeo se desarrolló y comenzó su evolución en el sector centro-oriental del Cinturón Volcánico Transmexicano (CVTM) ca. 7-8 Ma (Mioceno tardío) como resultado de fallas NESW (Israde-Alcantara et al., 2010; Olvera-García et al., 2020). Este se considera dentro de un sistema de cuenca de edad Neógena tardía al Reciente, que se extiende en varios bloques inclinados hacia el sur, delimitados por fallas hacia el norte que conforman varias cuencas de semigraben donde se desarrolló el actual lago (Israde-Alcantara y Garduño-Monroy, 1999).

El lago de Cuitzeo tiene un basamento conformado por lavas de composición andesítica que fueron emitidas hace $8 \pm 0.2 \mathrm{Ma}$ (Pola et al., 2016). La actividad tectónica produjo la fragmentación de estas 


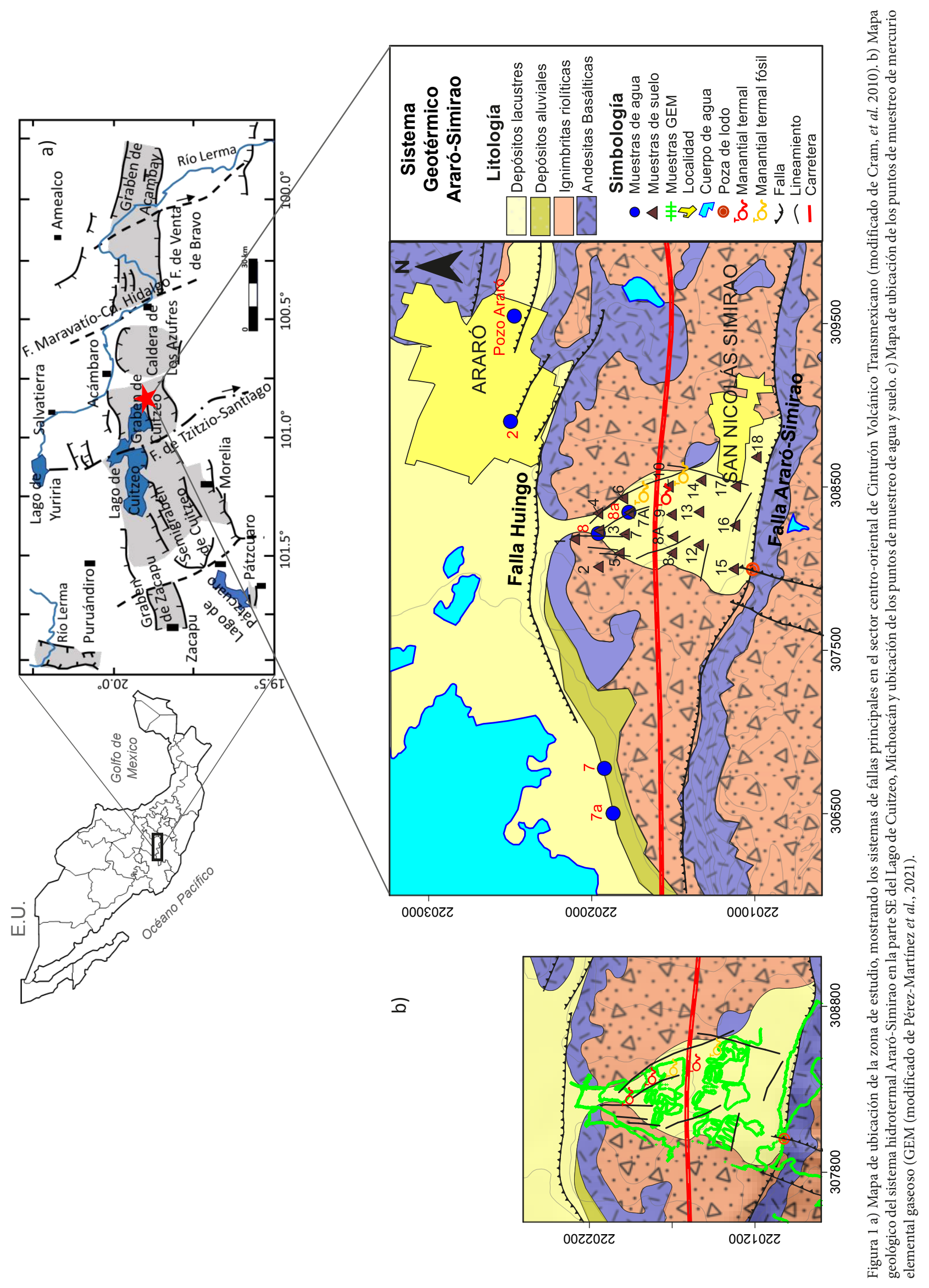


lavas generando un graben, que posteriormente fue rellenado por depósitos volcanosedimentarios (Israde-Alcantara et al., 2010). En el área se observan dos tipos de fallamiento, el más antiguo presenta una orientación NW-SE, afecta solo a rocas miocénicas y es paralelo a las estructuras originadas por la tectónica de Cuencas y Sierras que afecta al noroeste de México. El otro fallamiento está representado por un sistema de fallas normales con orientación de NE-SW que afectan a ignimbritas con edades de $2.8 \mathrm{Ma}$, mismas que presentan basculamiento de hasta $10^{\circ}$ (Garduño-Monroy y Gutiérrez -Negrín, 1992).

En el sistema geotérmico Araró-Simiro, las estructuras locales más importantes y aparentemente activas son las fallas de Huingo y Araró-Simirao (DETENAL, 1978), de tipo normal y orientación general ENW-WSE con variaciones en su rumbo a NE-SW y ENEWSW (Figura 1a). A través de ellas afloran manifestaciones termales (Olvera-García et al., 2020). Estas estructuras configuran un sistema de bloques escalonados hacia el norte (y en menor grado hacia el noroeste), con ligeros basculamientos hacia el sur (y en menor grado hacia el sureste) (Viggiano-Guerra y Gutiérrez Negrín, 2003; Olvera-García et al., 2020). Las unidades volcánicas de base a techo incluyen: andesitas, andesitas basálticas, riolitas vítreas, riolitas vítreas con textura fluidal, ignimbritas riolíticas, basaltos y depósitos de escoria. Estas unidades se encuentran cubiertas por depósitos de talud, así como aluviones. En el área de estudio afloran tres unidades: andesitas basálticas, riolitas vítreas e ignimbritas riolíticas, además de depósitos aluviales (Figura 1) (Viggiano-Guerra y Gutiérrez Negrín, 2003; Viggiano-Guerra y Gutiérrez Negrín, 2005; Pola et al., 2016).

Los suelos que predominan en el área de estudio son del tipo gleysol, los cuales se encuentran en zonas de inundación en la ribera del lago y pueden estar saturados por tiempo prolongado. Los suelos de este tipo presentan evidencias de procesos bajo condiciones reductoras por estar saturados con agua freática, muestran características de hidromorfismo y fangosidad, valores de $\mathrm{pH}$ relativamente ácidos a neutros y son importantes amortiguadores de los cuerpos de agua (Cram et al., 2010). En el área de estudio, el suelo del tipo gleysol se ubica en la zona de manifestaciones termales del sistema hidrotermal Araró-Simirao. Alrededor de la zona de manifestaciones termales, el suelo predominante es el vertisol; este tipo de suelo se localiza en planicies con alto contenido de arcillas expandibles (30\%) provocando la formación de grietas, son suelos profundos y susceptibles al anegamiento, con valores de $\mathrm{pH}$ de neutros a básicos (Cram, et al. 2010).

\section{Manifestaciones termales en el área de estudio}

La cuenca del lago de Cuitzeo se distingue por exhibir altos valores de flujo de calor ( $<200 \mathrm{mWm}^{2}$, Prol-Ledesma et al., 2018), y es por lo tanto una de las regiones con mayor flujo de calor e interés con fines exploratorios para producción eléctrica dentro de la Provincia Geotérmica del Cinturón Volcánico Transmexicano (Viggiano-Guerra y Gutiérrez Negrín, 2005; Prol-Ledesma y Morán-Zenteno, 2019). De acuerdo con Viggiano-Guerra y Gutiérrez Negrín (2005), el sistema geotérmico Araró-Simirao parece ser un reservorio de zona de fractura alimentado por un conducto profundo y estrecho relacionado con la falla Araró-Simirao. Es probablemente un sistema de convección, líquido-dominante de media a alta temperatura $\left(160^{\circ}\right.$ a $220^{\circ} \mathrm{C}$; Segovia et al., 2005a, 2005b, 2010) con aguas sódico-cloruradas (Viggiano-Guerra y Gutiérrez-Negrín, 2003). Por debajo de la zona hidrotermal de San Nicolás Simirao (Figura 1b) parece haber un lecho de sedimentos lacustres que actúa como un caprock, en donde las fallas y fracturas que cruzan con este lecho permiten la mezcla de fluidos geotérmicos subterráneos con el agua del acuífero dando lugar a depósitos en superficie de sínter, calcita y halita. El área hidrotermal de San Nicolás Simirao parece ser la única zona de ascenso del fluido desde la profundidad en toda la zona de Araró. Por otra parte, hacia el sureste de Araró se localiza el segundo campo geotérmico más importante de México, Los Azufres; además, aproximadamente a $2 \mathrm{~km}$ al noroeste, se localiza la zona de San Agustín del Maíz, donde se ha reportado actividad hidrotermal con temperaturas de descarga de $89^{\circ} \mathrm{C}$, una temperatura estimada con geotermometría de sílice de $195^{\circ} \mathrm{C}$ y con índice de saturación de aproximadamente $250{ }^{\circ} \mathrm{C}$ (Segovia et al., 2005a, 2005b). Un sistema geotérmico de características geológicas similares a la zona de estudio es el de San Bartolomé de los Baños en Guanajuato (Canet et al., 2019) al NE de Araró.

\section{METODOLOGÍA}

\section{Muestreo, análisis químico y mineralógico de suelos}

Se realizó el muestreo de suelo en la zona del sistema geotérmico Araró-Simirao (Figura 1b), a juicio de experto (mallado sobre la zona) y desfasado entre líneas de muestreo (tipo tresbolillo). Se colectaron 18 muestras en toda la zona de alteración hidrotermal (desde la falla Huingo al norte, hasta la falla Araró-Simirao al sur), a una profundidad de entre 5 y $15 \mathrm{~cm}$; las muestras fueron tamizadas a través de una malla de $2 \mathrm{~mm}$ para quitar materia orgánica, como raíces y plantas, y fragmentos con tamaños de grano por arriba de arenas gruesas, y se almacenaron en bolsas de plástico para su posterior análisis químico. En el laboratorio fueron extendidas por un periodo de $72 \mathrm{~h}$ a temperatura ambiente para eliminar su contenido de humedad. Para su análisis químico, los suelos se sometieron a la técnica de digestión ácida con el fin de disolver la matriz del suelo y solubilizar los metales en el laboratorio de Aerosoles Atmosféricos, Centro de Ciencias de la Atmósfera, de la Universidad Nacional Autónoma de México (UNAM). El procedimiento fue pesar $0.5 \mathrm{~g}$ de muestra de suelo, se le añadieron $10 \mathrm{~mL}$ de $\mathrm{HNO}_{3} 3.2 \mathrm{M}$ y $5 \mathrm{~mL}$ de $\mathrm{H}_{2} \mathrm{O}_{2} 32 \%$ (v/v); se llevó a cabo la reacción en tubos de teflón sellados en un sistema de digestión por microondas (Microwave Mars 5) por 20 min a $180^{\circ} \mathrm{C}, 250$ psi y $1200 \mathrm{~W}$; después de la digestión, las muestras fueron colocadas en reposo durante otras $24 \mathrm{~h}$, los vasos se despresurizaron y la solución fue decantada dentro de matraces aforados de $50 \mathrm{~mL}$, a los cuales se les adicionó agua desionizada del Tipo II ASTM, hasta la marca de aforo. Las muestras fueron colocadas en contenedores de polietileno hasta su posterior análisis. Todos los materiales usados durante el proceso de digestión fueron previamente lavados con ácido.

Para el análisis de la concentración de mercurio $(\mathrm{Hg})$, se tomó un volumen de $20 \mathrm{~mL}$ de las muestras de suelo procesadas por digestión ácida, se añadió $1.5 \mathrm{~mL}$ de $\mathrm{HNO}_{3}(3.2 \mathrm{M}), 1.25 \mathrm{~mL}$ de $\mathrm{KMnO}_{4}(5 \%)$. La cuantificación de $\mathrm{Hg}$ se realizó por Espectroscopia de Absorción Atómica, con un equipo GBC modelo 932 acoplado a un generador de hidruros por el método de vapor frío (GBC 932 AA, HG3000), utilizando una lámpara de catado hueco de $\mathrm{Hg}$ marca Photronlamps (Longitud de onda $253.7 \mathrm{~nm}$ ) y argón de ultra-alta pureza. El análisis químico de $\mathrm{As}, \mathrm{Cu}, \mathrm{V}$ y $\mathrm{Zn}$ se realizó de acuerdo con el método EPA 200.7, empleando un Espectrofotómetro de Emisión Óptica con Plasma Acoplado Inductivamente (ICP-OES) marca Thermo Fisher Scientific, modelo iCAP 7000. Los estándares de calibración fueron elaborados a partir de soluciones Inorganic Ventures de 1000 ppm en siete diferentes concentraciones; para Hg se realizó empleando un estándar certificado de mercurio marca High Purity (Hg-1000 mg/L). Los coeficientes de correlación para las curvas de calibración fueron mayores a 0.999 , los porcentajes de recuperación fueron superiores al $90 \%$ y fueron evaluados mediante un material de referencia estándar de High-Purity Standards (NIST- Nacional Institute of Standard and Technology de los Estados Unidos). El límite de detección (LD) del equipo ICP-OES fue: $5 \mu \mathrm{g} / \mathrm{L}(\mathrm{As}), 0.4 \mu \mathrm{g} / \mathrm{L}(\mathrm{Cu}), 0.6 \mu \mathrm{g} / \mathrm{L}(\mathrm{V})$ y $0.7 \mu \mathrm{g} / \mathrm{L}(\mathrm{Zn})$. Se utilizaron 
blancos de reactivos $\left(\mathrm{HNO}_{3}\right.$ y agua desionizada ASTM grado Tipo II). Además, se realizó el análisis mineralógico de 10 muestras seleccionadas de suelo, a través de microscopía electrónica y análisis de química mineral semicuantitativa, mediante un microscopio electrónico ambiental TM-1000 Hitachi con un detector acoplado de energía dispersiva de rayos X (EDX) del Laboratorio de Petrografía y Microtermometría de Inclusiones Fluidas del Instituto de Geofísica, UNAM. Para su análisis, las muestras fueron cuarteadas, tamizadas hasta malla 100 ASTM y colocadas entre dos portaobjetos de vidrio de dimensiones convencionales y recubiertas con grafito para la obtención de imágenes de electrones.

\section{Muestreo y análisis químico de agua}

Se colectaron 5 muestras de agua superficial de los manantiales termales (Balnearios Manantiales Curativos, La Playa, Huingo y Los Hervideros) y una muestra de agua de pozo (agua potable de la comunidad de Araró), tomadas a la descarga. Las muestras fueron filtradas (filtros de celulosa de tamaño de poro de $0.45 \mu \mathrm{m}$ ), acidificadas (ácido nítrico ultrapuro hasta un $\mathrm{pH}$ aproximado de 2) y almacenadas en envases de polietileno de alta densidad de $60 \mathrm{ml}$ (previamente lavados con $\mathrm{HNO}_{3}$ y $\left.\mathrm{HCl}\right)$ y puestas en refrigeración $\left(2{ }^{\circ} \mathrm{C}\right)$ hasta su análisis químico en laboratorio. En campo se determinaron los siguientes parámetros fisicoquímicos: $\mathrm{pH}$ (Standard Methods 4500-H+), temperatura (EPA 170.1), conductividad eléctrica y sólidos totales disueltos (Standard Methods 2510), potencial redox (Standard Methods 2580) con una sonda multiparamétrica (Aquatroll 600), cuyos electrodos fueron previamente verificados con soluciones de conductividad, solución Zobell y amortiguadores de pH. Además, se cuantificó el contenido de sulfuro de hidrógeno mediante la formación del complejo azul de metileno (Standard Methods $4500-S^{2-} \mathrm{D}$ ). Los análisis químicos se realizaron conforme a los Standard Methods for Examination of Water and Wastewater (APHA, 1998). La cuantificación de Hg se realizó por Espectroscopia de Absorción Atómica con un equipo GBC modelo 932 acoplado a un generador de hidruros por el método de vapor frío (HG3000) en el laboratorio de Aerosoles Atmosféricos, Centro de Ciencias de la Atmósfera, UNAM.

\section{Determinación del mercurio elemental gaseoso}

Los datos de mercurio elemental gaseoso (GEM por sus siglas en inglés: gaseous elemental mercury) se tomaron de Pérez-Martínez et al. (2021). Ellos realizaron un total de 18500 mediciones de la concentración de GEM, en la zona de estudio (Figura 1c). Las mediciones se obtuvieron con un analizador portátil de vapor de Hg Lumex-RA 915+, que es un espectrómetro de absorción atómica multifuncional portátil con corrección Zeeman para la absorción de fondo, que elimina el efecto de las impurezas interferentes. La medición consistió en el bombeo de aire ambiental de $10 \mathrm{dm}^{3} / \mathrm{min}$, aproximadamente a un metro del suelo, a través de una entrada que filtra las partículas del aire a la celda de detección. Durante el muestreo, cada 20 min se aplica una corrección de cero para restablecer la línea de base, cambiando el flujo de aire hacia un filtro de absorción del mercurio. El rango de medición del equipo es de $0.5-30000 \mathrm{ng} / \mathrm{m}^{3}$, y el instrumento tiene una precisión del $20 \%$. Posteriormente se realizó un análisis de estadística básica (p. ej., promedio, máximo, mínimo) para el manejo de los datos.

\section{Elaboración de diagramas de Pourbaix y de solubilidad}

Para identificar los procesos de transporte y movilidad del mercurio, se empleó la modelación química para estimar las especies químicas presentes en el agua bajo las condiciones fisicoquímicas determinadas en campo. Se empleó el programa The Geochemist's Workbench (GWB) versión 11.0, específicamente los módulos: GSS (para calcular la actividad de las especies químicas de $\mathrm{Hg}, \mathrm{SO}_{4}{ }^{2-} \mathrm{y} \mathrm{Cl}$ ), y el módulo Act2 para la elaboración del diagrama de Eh vs $\mathrm{pH}$. Para la elaboración de los diagramas de mercurio se requiere una base de datos que incluya complejos acuosos de mercurio, por tal motivo se generó una nueva base de datos con información termodinámica a partir de las bases Thermo (complejos sulfurados) y Thermo. Minteq (complejos clorurados) que están almacenadas en el programa de GWB.

\section{RESULTADOS}

\section{Caracterización de la química de agua}

En la Tabla 1 se muestran las concentraciones de iones mayores, $\mathrm{S}^{2}$ y Hg en las muestras de agua, así como los valores obtenidos de los parámetros fisicoquímicos medidos en campo. El rango de temperaturas varía de 31 a $76^{\circ} \mathrm{C}$, por lo que casi todas las muestras se pueden considerar termales (temperatura de la descarga $5^{\circ} \mathrm{C}$ por arriba de la temperatura máxima promedio anual de $29.7^{\circ} \mathrm{C}$ para la zona de estudio; SMN, 2019), excepto para la muestra LSA- 02 cercana al poblado de Araró, la cual es una descarga fría. De acuerdo con el pH se consideran aguas neutras a ligeramente básicas. Derivado de los valores de conductivi$\mathrm{dad}$, todas las muestras representan aguas potables $(\mathrm{CE}<2500 \mu \mathrm{S} / \mathrm{cm})$, excepto las muestras LSA-08 y LSA-08A que son aguas salobres (Figura 2a), las cuales tienen también temperaturas altas $\left(76^{\circ} \mathrm{C}\right)$; la misma tendencia se observa para el contenido de sólidos totales disueltos (STD). Las muestras presentan valores de potencial óxidoreducción (Eh) en un rango de 293 a $362 \mathrm{mV}$, indicando condiciones oxidantes. En cuanto al contenido de iones mayores (Tabla 1), las aguas

Tabla 1. Parámetros fisicoquímicos y química (iones mayores, menores y elementos traza) de muestras de agua de la zona geotérmica de Araró-Simirao. T.A.: temperatura ambiental T.D.: temperatura a la descarga de las manifestaciones y pozo. CE: conductividad eléctrica, STD: sólidos totales disueltos, Eh: potencial redox, B. I.: balance iónico.

\begin{tabular}{|c|c|c|c|c|c|c|c|c|c|c|c|c|c|c|c|c|c|}
\hline \multirow[t]{2}{*}{ Muestra } & \multirow{2}{*}{$\begin{array}{l}\text { T.A. } \\
{ }^{\circ} \mathrm{C}\end{array}$} & \multirow{2}{*}{$\begin{array}{c}\text { T.D. } \\
{ }^{\circ} \mathrm{C}\end{array}$} & \multirow[t]{2}{*}{$\mathrm{pH}$} & \multirow{2}{*}{$\begin{array}{c}\text { CE } \\
\mu \mathrm{S} / \mathrm{cm}\end{array}$} & \multirow{2}{*}{$\begin{array}{l}\text { STD } \\
\mathrm{mg} / \mathrm{L}\end{array}$} & \multirow{2}{*}{$\begin{array}{l}\text { Eh } \\
\mathrm{mV}\end{array}$} & $\mathrm{Na}^{+}$ & $\mathbf{K}^{+}$ & $\mathrm{Mg}^{2+}$ & $\mathrm{Ca}^{2+}$ & $\mathrm{Cl}^{-}$ & $\mathrm{SO}_{4}{ }^{2-}$ & $\mathrm{NO}_{3}{ }^{-}$ & $\mathrm{HCO}_{3}^{-}$ & \multirow{2}{*}{$\begin{array}{c}\text { B.I. } \\
\%\end{array}$} & \multirow{2}{*}{$\begin{array}{c}\mathbf{S}^{-2} \\
\mathrm{mg} / \mathrm{L}\end{array}$} & \multirow{2}{*}{$\begin{array}{r}\mathbf{H g} \\
\mu \mathrm{g} / \mathrm{L}\end{array}$} \\
\hline & & & & & & & \multicolumn{8}{|c|}{$\mathrm{mg} / \mathrm{L}$} & & & \\
\hline LSA-02 & 30 & 31 & 7.1 & 353 & 204 & 362 & 133.40 & 33.16 & 2.84 & 36.48 & 200.81 & 206.12 & 26.34 & 114.56 & -8.7 & 0.02 & 3.60 \\
\hline LSA-07 & 25 & 41 & 6.9 & 2965 & 1460 & 347 & 318.05 & 28.55 & 2.17 & 36.84 & 462.92 & 79.31 & 5.14 & 171.88 & 0.1 & 0.02 & 4.45 \\
\hline LSA-07A & 25 & 39 & 7.3 & 1860 & 940 & 304 & 200.02 & 19.88 & 2.12 & 20.81 & 291.78 & 63.34 & 5.47 & 164.51 & -6.0 & 0.02 & 1.45 \\
\hline LSA-08 & 30 & 76 & 8.1 & 5916 & 3109 & 293 & 744.74 & 50.81 & BLC & 41.09 & 927.20 & 192.80 & BLC & 82.12 & 10.2 & 0.02 & 1.09 \\
\hline LSA-08A & 31 & 76 & 7.1 & 5651 & 3246 & 362 & 719.81 & 48.37 & BLC & 44.37 & 929.45 & 217.19 & BLC & 98.93 & 6.8 & 0.02 & 9.96 \\
\hline \multirow[t]{2}{*}{ Pozo Araró } & 29 & 38 & 7.5 & 176 & 99 & 343 & 21.16 & 7.72 & 4.37 & 18.20 & 8.13 & 8.33 & 1.02 & 117.48 & 2.7 & 0.10 & 2.29 \\
\hline & & & & & & $\mathrm{LC}$ & 0.04 & 0.40 & 0.20 & 0.20 & 0.80 & 1.10 & 0.1 & & & 0.01 & 0.45 \\
\hline
\end{tabular}

LC: límite de cuantificación. BLC: valores por debajo del límite de cuantifiación. 


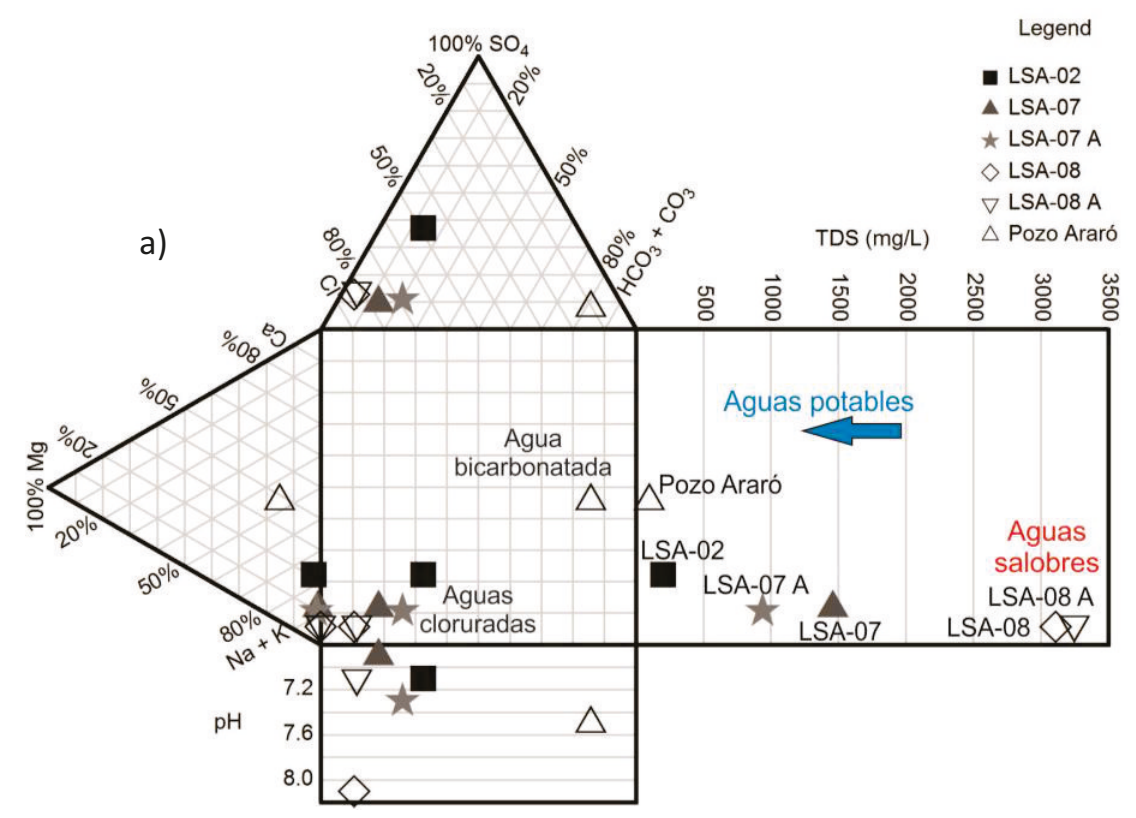

$\mathrm{Na}^{+}-\mathrm{Cl}^{-}$

b)

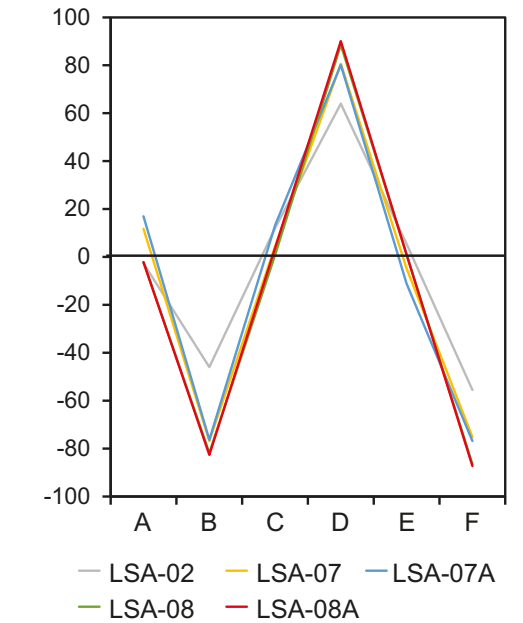

$\mathrm{Ca}^{2+}-\mathrm{Na}^{+}-\mathrm{HCO}_{3}^{-}$

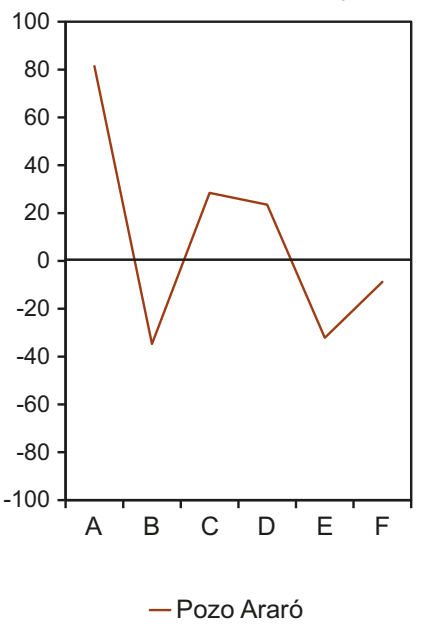

Figura 2. Diagramas de (a) Durov que muestra el tipo de agua de acuerdo con la concentración relativa de los iones mayoritarios y sus características fisicoquímicas (STD, CE y pH), y (b) D’Amore mostrando los dos tipos de agua, sódico-clorurada $\left(\mathrm{Na}^{+}-\mathrm{Cl}-\right)$ y bicarbonatada mixta $\left(\mathrm{Ca}^{2+}-\mathrm{Na}^{+}-\mathrm{HCO}_{3}^{-}\right)$, de acuerdo con su contexto geológico.

presentan altas concentraciones de $\mathrm{Na}^{+}$y $\mathrm{K}^{+}$así como de $\mathrm{Cl}^{-}$y $\mathrm{SO}_{4}{ }^{2-}$, y bajas concentraciones de $\mathrm{Ca}^{2+}, \mathrm{Mg}^{2+}$ así como de $\mathrm{HCO}_{3}{ }^{-}$y $\mathrm{NO}_{3}$. De acuerdo con el diagrama de Durov (Figura 2a) existen dos facies hidrogeoquímicas principales por la predominancia de iones: (1) aguas sódico-cloruradas (LSA-08, LSA-08A, LSA07 y LSA-07A) y con ligero enriquecimiento en sulfatos (LSA-02), y (2) agua bicarbonatada mixta (pozo Araró). La primera facies hidrogeoquímica se corresponde con las muestras termales y la segunda se corresponde con la muestra del pozo de agua potable. De acuerdo con el diagrama de clasificación de D’Amore (D'Amore et al., 1983) (Figura 2b), que considera el contexto geológico, las aguas del tipo 1 se clasifican como aguas de circulación profunda a través de un basamento cristalino y el agua del tipo 2 como agua que ha circulado por terrenos sedimentarios arcillosos.

De acuerdo con Custodio y Llamas (1996), las aguas subterráneas, en general, tienen concentraciones de elementos traza menores a $1 \mu \mathrm{g} / \mathrm{L}$; sin embargo, las muestras de agua de la zona de estudio presentan concentraciones anómalas de Hg (Tabla 1). Si comparamos estas con- centraciones de $\mathrm{Hg}$ con las establecidas en la normatividad mexicana para aguas de uso y consumo humano (NOM-127-SSA-1994), todas las muestras sobrepasan el límite máximo permisible de $\mathrm{Hg}(1 \mu \mathrm{g} / \mathrm{L})$. Se ha reportado que la concentración de mercurio es mayor en aguas termales (p. ej., $0.5-4 \mu \mathrm{g} / \mathrm{L}$ en manantiales termales de Kamchatka, Rusia; USGS, 1970), lo cual es consistente con los resultados que se muestran en la Tabla 1: la muestra que presenta el mayor contenido de $\mathrm{Hg}(9.96 \mu \mathrm{g} / \mathrm{L}$; LSA-08A) se corresponde con el manantial de mayor temperatura $\left(76^{\circ} \mathrm{C}\right)$ en la zona de estudio.

\section{Mineralogía y contenido de $\mathrm{Hg}$ en suelos}

Los principales minerales encontrados en las muestras de suelos son aluminosilicatos (feldespato potásico y plagioclasa), además de óxidos de Fe, ópalo A y hornblenda (Figura 3b); estos minerales se encontraron sobre todo en la zona norte del sistema (zona Araró). Por otra parte, en la zona sur (San Nicolás Simirao) se encontraron, además, arsenopirita y otros minerales de procedencia hidrotermal 

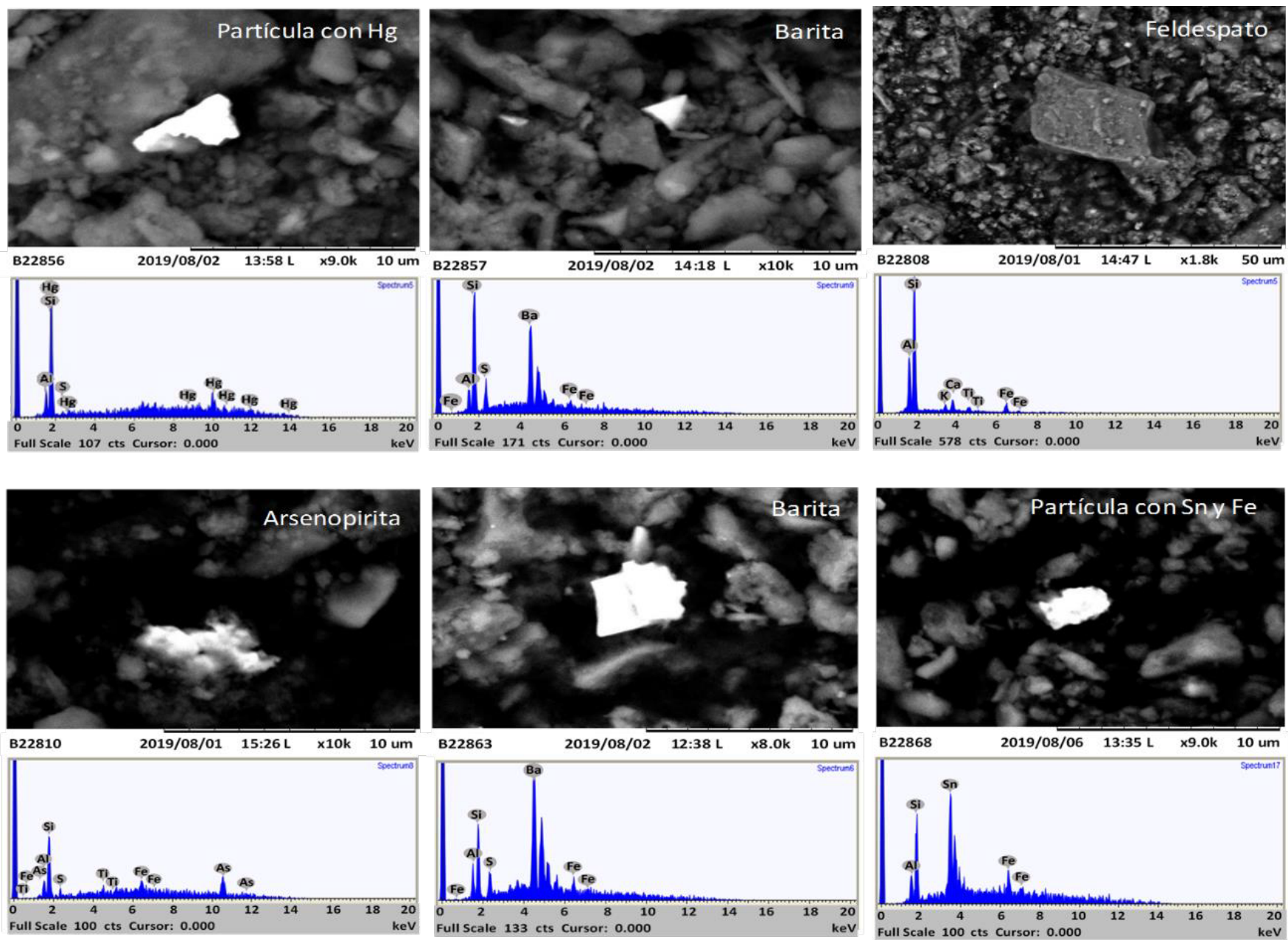

Figura 3a. Imágenes de microscopia electrónica y análisis de química semicuantitativa EDX de suelos seleccionados en la zona de San Nicolás Simirao.

como la barita (muestras cercanas a la falla Araró-Simirao), partículas con Fe y Sn, y partículas con Hg (muestra LS-S-14) (Figura 3a). De acuerdo con los análisis químicos (Tabla 2), los suelos presentan altos contenidos de As entre 172 y $1218 \mu \mathrm{g} / \mathrm{g}$, y en menor proporción de $\mathrm{Cu}$ $(11-28 \mu \mathrm{g} / \mathrm{g})$ y Mn $(12-33 \mu \mathrm{g} / \mathrm{g})$; las menores concentraciones se obtuvieron para $\mathrm{Hg}(0.07-21 \mu \mathrm{g} / \mathrm{g}), \mathrm{Zn}(0.8-4 \mu \mathrm{g} / \mathrm{g})$ y V $(0.22-3 \mu \mathrm{g} / \mathrm{g})$. La presencia de algunos de los elementos analizados concuerda con la mineralogía encontrada en los suelos como la arsenopirita. Las concentraciones de $\mathrm{Hg}$ obtenidas son similares a las reportadas por Phuong et al. (2012) para los suelos del sistema geotérmico Ungaran en Indonesia (0 - 21.56 ppm); en la zona de estudio la muestra con la concentración más elevada de Hg es la LS-S-14, cuya mineralogía muestra partículas con $\mathrm{Hg}$, y se colectó en un área cercana a un manantial termal fósil del poblado San Nicolás Simirao (Figura 1b).

\section{Distribución de $\mathrm{Hg}$ en agua y suelo}

En la Figura 4 se muestra el mapa de distribución de concentraciones de $\mathrm{Hg}$ en el agua; se observa que la muestra con mayor contenido de $\mathrm{Hg}(9.96 \mu \mathrm{g} / \mathrm{L}$, manantial de mayor temperatura, muestra LSA-08A) se ubica sobre uno de los lineamientos descritos por Ávalos-Tapia (2016), con dirección NW-SE, que es el mismo en donde se ubican varios de los manantiales termales activos y fósiles de la zona cercana al poblado de Araró. También se observa alto contenido en $\mathrm{Hg}$ en un manantial ubicado en la parte oeste de la zona ( $4.45 \mu \mathrm{g} / \mathrm{L}$, muestra LSA-07), que
Tabla 2. Concentración de metales determinados en muestras de suelo de la zona geotérmica de Araró-Simirao.

\begin{tabular}{lrrrrrr}
\hline \multirow{2}{*}{ Muestra } & \multicolumn{1}{c}{ As } & Hg & \multicolumn{1}{c}{ Cu } & Mn & V & Zn \\
\cline { 2 - 7 } & \multicolumn{5}{c}{$(\mu \mathrm{g} / \mathrm{g})$} & \\
\hline LS-S-01 & 266.46 & 0.17 & 15.52 & 16.05 & 0.36 & 0.80 \\
LS-S-02 & 176.55 & 0.35 & 20.83 & 22.99 & 0.33 & 2.46 \\
LS-S-03 & 240.34 & 0.59 & 11.77 & 12.76 & 0.57 & 4.64 \\
LS-S-04 & 172.99 & 0.07 & 22.16 & 22.61 & 0.53 & 1.36 \\
LS-S-05 & 338.75 & 0.42 & 18.43 & 18.92 & 0.59 & 1.13 \\
LS-S-06 & 187.76 & 0.28 & 25.03 & 25.53 & 0.43 & 1.59 \\
LS-S-07 & 399.74 & 0.24 & 16.68 & 21.42 & 3.15 & 1.96 \\
LS-S-07a & 249.25 & 0.24 & 13.03 & 14.97 & 2.02 & 1.59 \\
LS-S-08 & 1218.45 & 0.15 & 28.57 & 33.67 & 3.02 & 2.18 \\
LS-S-08a & 286.79 & 0.16 & 12.31 & 13.43 & 0.22 & 0.98 \\
LS-S-09 & 264.85 & 0.24 & 13.08 & 13.75 & 0.86 & 1.76 \\
LS-S-10 & 678.00 & 0.51 & 23.23 & 23.82 & 0.45 & 0.94 \\
LS-S-12 & 253.28 & 1.24 & 12.40 & 21.21 & 2.53 & 1.12 \\
LS-S-13 & 210.04 & 1.88 & 20.83 & 22.99 & 0.33 & 2.46 \\
LS-S-14 & 1031.16 & 21.04 & 11.77 & 12.76 & 0.57 & 4.64 \\
LS-S-15 & 224.63 & 3.12 & 22.16 & 22.61 & 0.53 & 1.36 \\
LS-S-16 & 338.75 & 1.68 & 18.43 & 18.92 & 0.59 & 1.13 \\
LS-S-17 & 187.76 & 4.38 & 25.03 & 25.53 & 0.43 & 1.59 \\
LS-S-18 & 399.74 & 1.17 & 16.68 & 21.42 & 3.15 & 1.96 \\
\hline
\end{tabular}



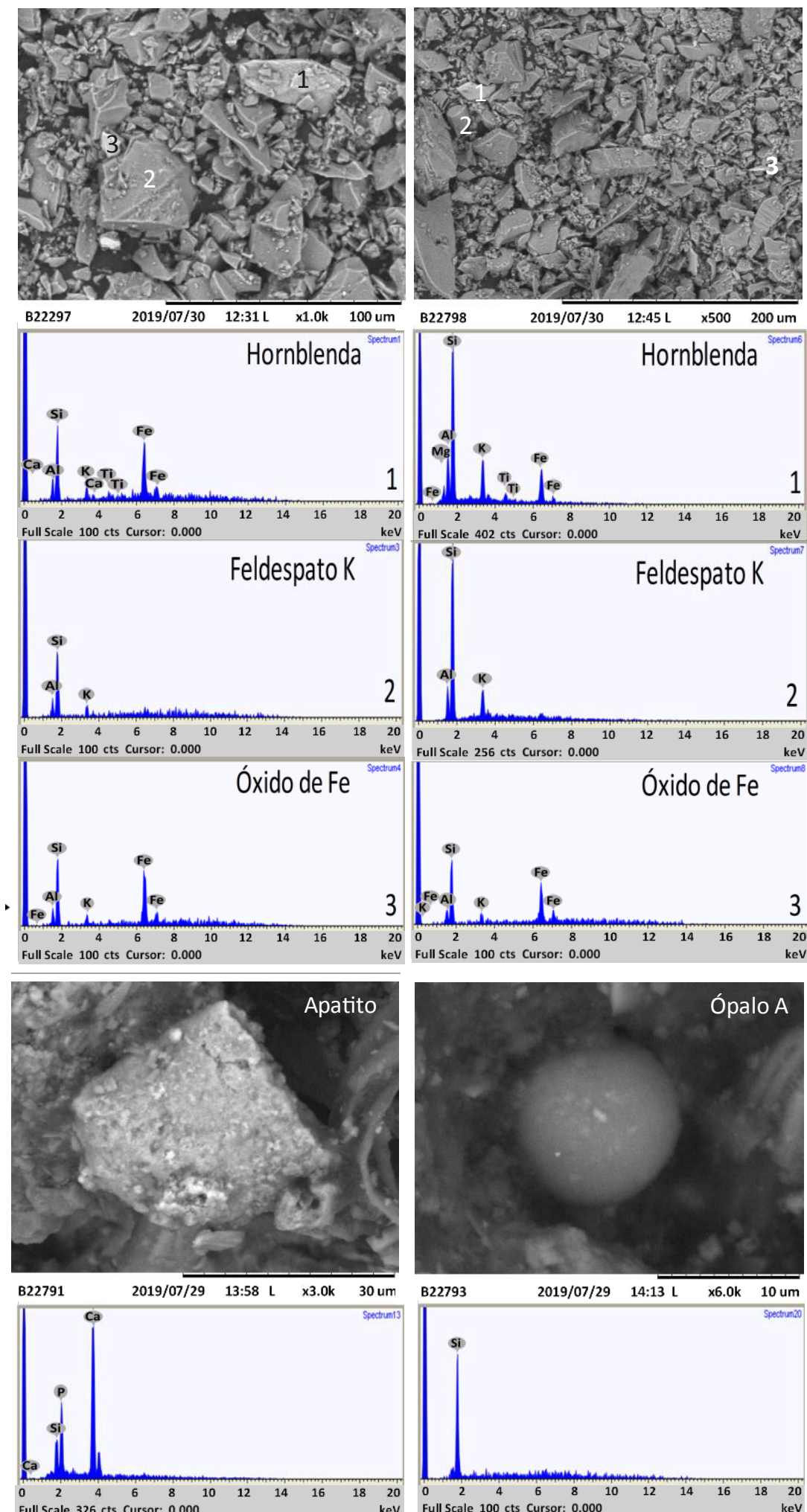

Full Scale 100 cts Cursor: 0.000
Figura 3b. Imágenes de microscopia electrónica y análisis de química semicuantitativa EDX de suelos seleccionados en la zona de Araró. podría estar relacionado con la falla de Huingo. Por otra parte, en el mapa de distribución de concentraciones de $\mathrm{Hg}$ en las muestras de suelo (Figura 5a), se observa que en la zona de San Nicolás Simirao (parte meridional de la zona de estudio) se encuentran las mayores concentraciones de $\mathrm{Hg}$ configurando dos aureolas de anomalías. La mayor anomalía se ubica en la parte SE asociada probablemente al alineamiento NW-SE y al manantial termal fósil cercano al poblado de San Nicolás Simirao; la otra se ubica en la parte SW, en el cruce de la falla Araró-Simirao con un alineamiento N-S en donde se ubica una poza de lodo con emanaciones de gases (Figura 1b). 


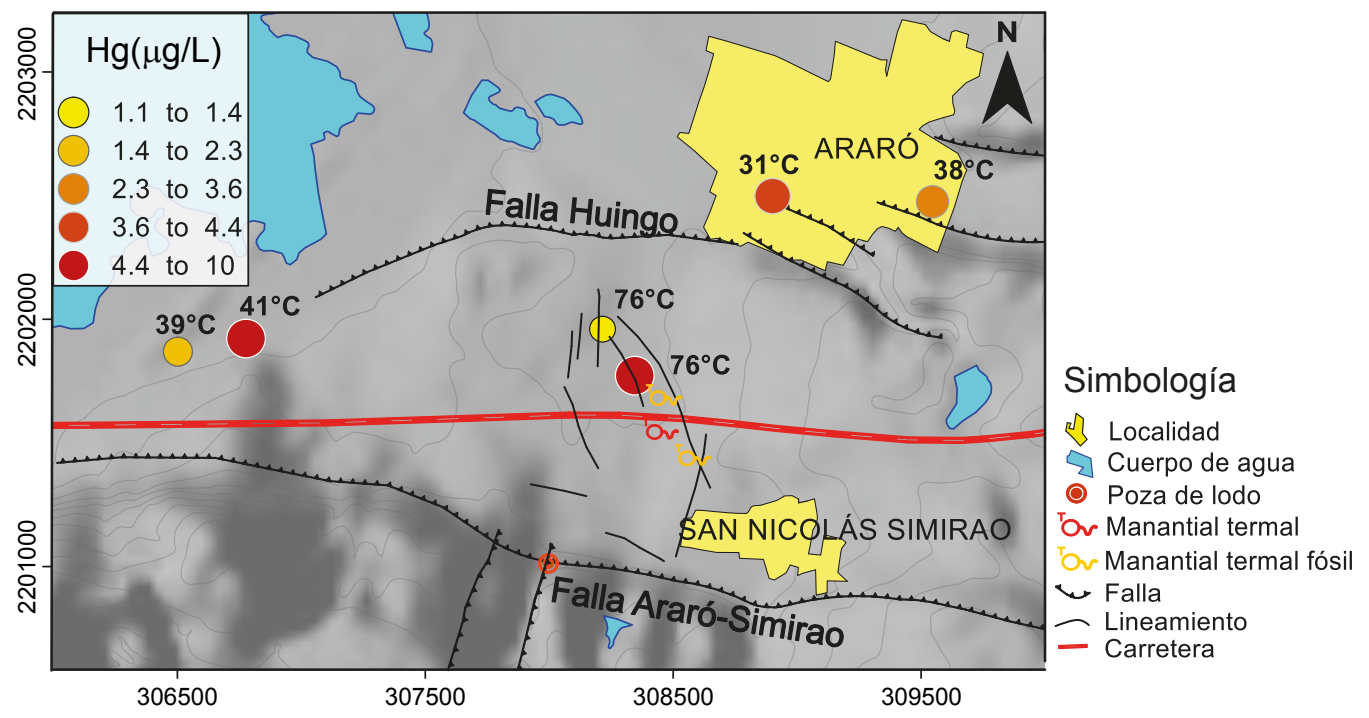

Figura 4. Mapa de distribución de la concentración de Hg en agua del sistema geotérmico Araró-Simirao.

\section{DISCUSIÓN}

\section{Distribución del mercurio}

Para poder relacionar el contenido de $\mathrm{Hg}$ en tres matrices diferentes (aire, agua y suelo) del sistema geotérmico estudiado, se compararon los datos de las concentraciones de mercurio elemental gaseoso (GEM) (Pérez-Martínez et al., 2021), cuyas concentraciones oscilaron en un rango de $0.5 \times 10^{-3}$ a $2.9 \mu \mathrm{g} / \mathrm{m}^{3}$ (Tabla 3 ), con las concentraciones de $\mathrm{Hg}$ en agua y suelos (Figura 4 y $5 \mathrm{a}$ ).

En la Figura 5a se muestran los mapas de distribución de las concentraciones de GEM y del Hg en suelos. Se observa que las mayores concentraciones de $\mathrm{Hg}$ tanto en el aire como en el suelo se ubican en la parte sur del sistema geotérmico, configurando dos aureolas de anomalías: una cercana a la falla de Araró y otra cercana al poblado de San Nicolás Simirao. Para la zona norte, las concentraciones de Hg son menores y solo se observa una pequeña anomalía de GEM en la zona de manantiales. Se realizaron perfiles de las concentraciones de GEM y Hg (sección A-A') a partir de la zona de mayor concentración con una orientación SE-NW (Figura 5b), derivado de la orientación que presentan los manantiales termales tanto activos como fósiles. En estos perfiles se observa que las anomalías de $\mathrm{Hg}$ en aire y suelo coinciden y se ubican en un área que corresponde con la zona de un manantial termal fósil cercano al poblado de San Nicolás Simirao, donde se reportan lineamientos con dirección SE-NW y NW-SE. Las concentraciones de $\mathrm{Hg}$ en el suelo decrecen en dirección NW sin volver a aumentar sobre del perfil. Las concentraciones de GEM decrecen en la parte intermedia del perfil, pero hacia la parte NW vuelven a aumentar en menor proporción, coincidiendo con la zona de manantiales termales activos de alta temperatura y donde se encontró la mayor concentración de $\mathrm{Hg}$ en agua (LSA-08A).

Tabla 3. Estadígrafos de las concentraciones de mercurio elemental gaseoso (GEM) en el sistema geotérmico de Araró-Simirao.

\begin{tabular}{lcccc}
\hline \multirow{2}{*}{ Puntos de muestreo } & Mínimo & Máximo & Promedio & Mediana \\
\cline { 2 - 5 } & \multicolumn{4}{c}{$\mu \mathrm{g} / \mathrm{m}^{3}$} \\
\hline 18500 & $0.5 \times 10^{-3}$ & 2.9 & $5 \times 10^{-3}$ & $2.6 \times 10^{-3}$ \\
\hline
\end{tabular}

De acuerdo con lo anterior, las anomalías de GEM a lo largo del perfil están relacionadas con el contenido de $\mathrm{Hg}$ en los suelos y aguas termales del sistema, siendo que para la zona sur se asocian con el alto contenido de $\mathrm{Hg}$ en el suelo, mientras que para la zona norte se asocian con las descargas de los manantiales termales de alta temperatura. Por otra parte, la anomalía de GEM encontrada en la falla Araró-Simirao se asocia con la salida directa de gases termales profundos a través de la traza de la falla.

\section{Geoquímica del mercurio}

De acuerdo con sus características químicas, el $\mathrm{Hg}$ presenta baja solubilidad y alta volatilidad aún a bajas temperaturas, por lo que los GEM pueden ser transportados en la atmósfera, (Lindqvist y Rodhe, 1985; Slemr y Scheel, 1985; Lindberg et al., 2007 Ariya et al., 2008; Bagnato et al., 2018) y depositados en el suelo por vía húmeda, o en menor proporción por vía seca (USEPA, 1997; Yu et al. 2018). Varekamp y Buseck (1983) observaron que el Hg originado en sistemas hidrotermales es retenido en los suelos en cantidades mayores a los valores de fondo en una zona sin actividad hidrotermal, aun considerando las variaciones locales de $\mathrm{Hg}$ (derivadas del cambio en el $\mathrm{pH}$, temperatura y humedad), que puedan estar asociadas con la retención en arcillas y materia orgánica. Por lo tanto, las anomalías de $\mathrm{Hg}$ encontradas en los suelos de la zona sur (San Nicolás Simirao) pueden estar asociadas directamente con el transporte del $\mathrm{Hg}$ proveniente del sistema hidrotermal a la superficie y no ser controladas por los parámetros antes mencionados.

Las altas concentraciones de $\mathrm{Hg}$ (hasta $9.96 \mu \mathrm{g} / \mathrm{L}$ ) encontradas en los manantiales con mayor temperatura, derivadas del ascenso de los fluidos geotérmicos desde el reservorio a la superficie, se deben a que gran cantidad de agua caliente con vapores de $\mathrm{Hg}$ puede fluir dentro del acuífero somero y salir a la superficie en manantiales calientes (Varekamp y Buseck, 1983). En ambientes termales, el contenido de Hg puede estar relacionado también con el transporte de cinabrio $(\mathrm{HgS}$; mineral de $\mathrm{Hg}$ más común) en soluciones acuosas como complejos de sulfuros o bisulfuros bajo condiciones geológicas de $100^{\circ} \mathrm{C}$ a por lo menos $200{ }^{\circ} \mathrm{C} \mathrm{y} \mathrm{pH}$ de neutro a alcalino (Barnes et al., 1967). El mercurio puede depositarse también como hidróxidos o carbonatos de mercurio, o incluso formar complejos solubles con ligantes del humus, debido a la gran cantidad de materia orgánica que contiene el suelo. 
a)
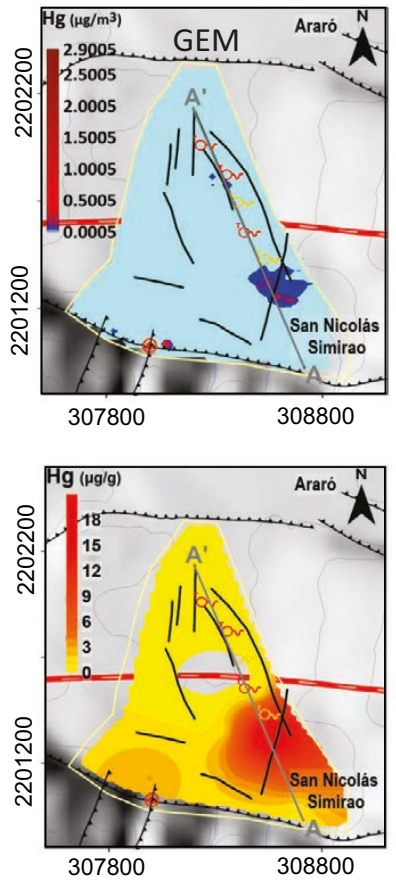

b)
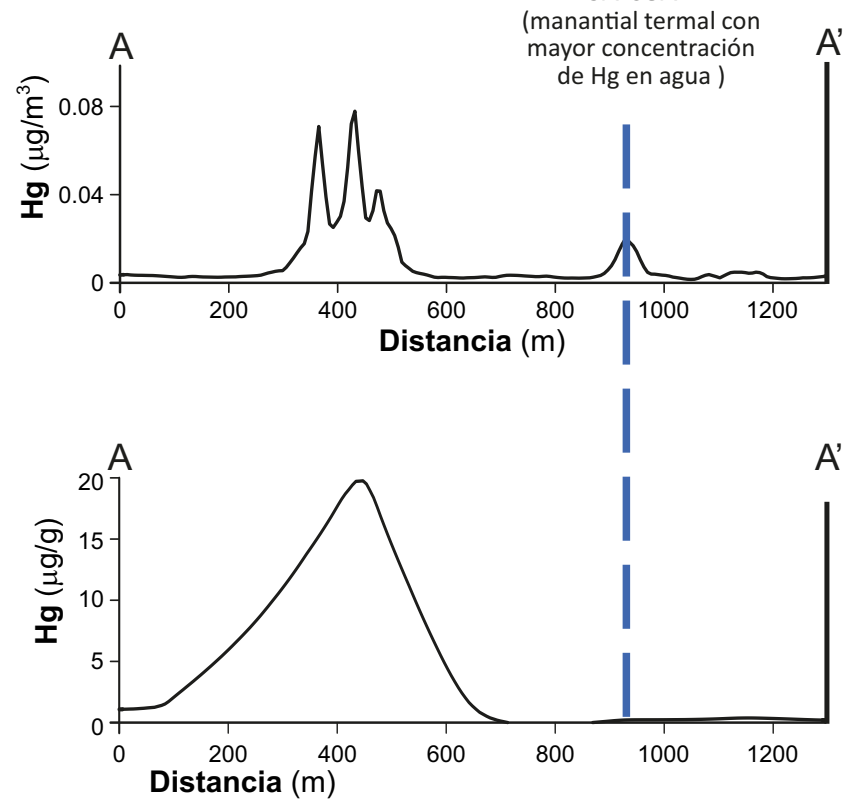

Figura 5. a) Mapa de distribución de la concentración en el aire de mercurio elemental gaseoso (GEM en $\mu \mathrm{g} / \mathrm{m}^{3}$; modificado de Pérez-Martínez et al., 2021) y mapa de distribución de la concentración de $\mathrm{Hg}$ en suelos (en $\mu \mathrm{g} / \mathrm{g}$ ), de la zona geotérmica Araró-Simirao. La mayor parte del área de muestreo presenta valores de GEM por debajo del valor umbral $\left(0.012 \mu \mathrm{g} / \mathrm{m}^{3}\right.$, zona azul claro) determinado para la zona de estudio, pero arriba del límite de detección del equipo $\left(0.0005 \mu \mathrm{g} / \mathrm{m}^{3}\right)$; b) Perfiles de concentración de GEM en el aire y del Hg en suelos, a lo largo de la sección A-A' mostrada en los mapas, junto con la ubicación del manantial termal con mayor contenido de Hg.

El mercurio también forma complejos inorgánicos estables con los iones cloruros como ligantes, y es a través de la formación de estos complejos que el mercurio se puede transportar en el agua. La predominancia de los complejos formados dependerá de la concentración de los sulfuros con respecto a los cloruros. En el caso de las aguas termales del presente trabajo, la concentración de bisulfuro es demasiado pequeña $(0.02 \mathrm{mg} / \mathrm{L}$ para la mayoría de las muestras y $0.1 \mathrm{mg} / \mathrm{L}$ para la muestra Pozo Araró; Tabla 1) con respecto a los cloruros. Por lo que, bajo las condiciones de $\mathrm{pH}(6.9$ a 8.1$)$ y Eh $(0.293$ a $0.362 \mathrm{v})$, la especie predominante es el $\mathrm{Hg}_{\text {(ac) }}^{0}$ (Figura 6) a través de la siguiente reacción de dismutación:

$$
\mathrm{Hg}_{2}{ }^{2+}{ }_{\text {(ac) }} \leftrightarrow \mathrm{Hg}^{0}{ }_{(\mathrm{ac})}+\mathrm{Hg}^{2+}{ }_{(\mathrm{ac})}
$$

Sin embargo, dadas las altas concentraciones de cloruros y altas temperaturas, la abundancia de $\mathrm{Hg}^{0}{ }_{\text {(ac) }}$ es menor con respecto a la de $\mathrm{Hg}^{2+}$ (Varekamp y Buseck, 1984). Por tanto, en soluciones con altos contenidos de cloruro (arriba de $35.45 \mathrm{mg} / \mathrm{L}$ ), la solubilidad del mercurio en aguas oxigenadas puede deberse a la presencia del complejo
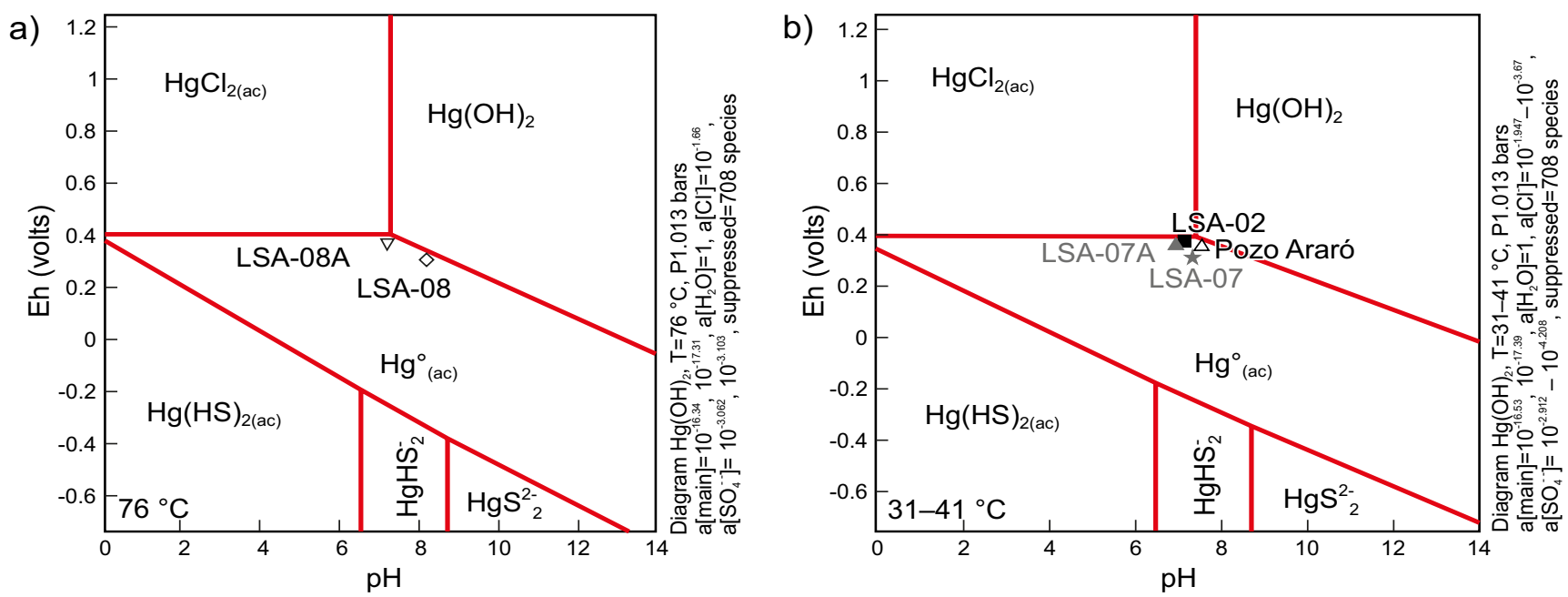

Figura 6. Diagramas de especies acuosas del mercurio. a) Para muestras de agua con temperatura a la descarga de $76^{\circ} \mathrm{C} \mathrm{y} \mathrm{b)} \mathrm{para} \mathrm{muestras} \mathrm{de} \mathrm{agua} \mathrm{con} \mathrm{temperatura}$ a la descarga de $31-41^{\circ} \mathrm{C}$. Cada diagrama se realizó bajo las condiciones fisicoquímicas determinadas en campo, así como con las actividades calculadas a partir de las concentraciones analíticas cuantificadas en laboratorio. 


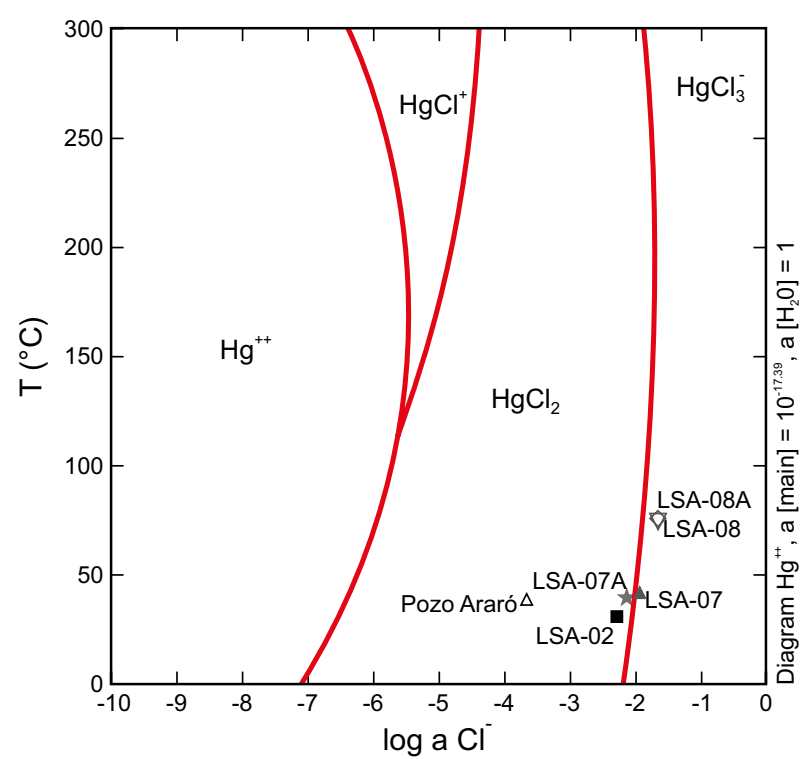

Figura 7. Diagrama del logartimo de la actividad de cloruros versus temperatura $\left({ }^{\circ} \mathrm{C}\right)$ donde se muestra la especiación de los complejos clorurados del mercurio en las aguas termales. Se muestra la ubicación de las muestras de agua de Araró en los campos correspondientes a las especies $\mathrm{HgCl}_{2}$ y $\mathrm{HgCl}_{3}{ }^{-}$.

químico $\mathrm{HgCl}_{2}$ (Hem, 1970). De acuerdo con el diagrama de solubilidad (Figura 7), las especies químicas predominantes en las muestras de agua de la zona de estudio son $\mathrm{HgCl}_{2}$ y $\mathrm{HgCl}_{3}{ }^{-}$. Sin embargo, una parte del mercurio $\mathrm{Hg}_{(\text {ac) }}^{0}$ tiende a ser volátil al estar expuesta la superficie del agua al aire (Hem, 1970).

\section{Modelo de transporte del $\mathrm{Hg}$}

En el modelo conceptual para el transporte de $\mathrm{Hg}$ en la zona geotérmica Araró-Simirao (Figura 8) se infiere que el Hg como gas elemental (GEM) es transportado a la superficie junto con los fluidos termales a través de zonas de mayor permeabilidad como la falla AraróSimirao, en donde existe ascenso de una fase de vapor enriquecida en $\mathrm{CO}_{2}$, así como también a través de fracturas reportadas en el área. El $\mathrm{Hg}$ se puede particionar dentro de esta fase de vapor desarrollándose una anomalía de $\mathrm{Hg}^{0}{ }_{\mathrm{g})}(\mathrm{GEM})$ en la zona de ascenso como es la zona de la poza de lodo y las áreas de manantiales termales.

Cuando el fluido geotérmico junto con vapores de $\mathrm{Hg}^{0}{ }_{(\mathrm{g})}$, probablemente derivados de magmas, llega al acuífero somero, éste se enriquece en $\mathrm{Hg}$, debido a procesos de intercambio iónico y adsorción del vapor (Varekamp y Buseck, 1983). Este proceso se refleja en una mayor concentración de $\mathrm{Hg}$ en el agua de los manantiales calientes, como ocurre en el manantial de mayor temperatura (LSA-08A) de la zona (a excepción de la muestra LSA-08 con alta temperatura pero con bajo contenido de $\mathrm{Hg}$, debido probablemente a la presencia de materia orgánica en el manantial). Dentro del acuífero, $\mathrm{el} \mathrm{Hg}^{0}{ }_{\text {(ac) }}$ sufre procesos de oxidación formándose el ión $\mathrm{Hg}^{2+}$. Las especies predominantes en la fase acuosa son el ion mercúrico $\left(\mathrm{Hg}^{2+}\right)$ y $\mathrm{Hg}_{(\mathrm{ac}}{ }^{2}$, los cuales provienen de la dismutación del ion mercuroso $\left(\mathrm{Hg}_{2}{ }^{2+}\right)$. El ion mercúrico forma complejos clorurados representados por las especies químicas: $\mathrm{HgCl}_{2}$ y $\mathrm{HgCl}_{3}^{-}$, los cuales transportan al mercurio en la fase acuosa. Posteriormente, los complejos de $\mathrm{Hg}$ son transportados en la dirección de flujo del acuífero somero generando una aureola de anomalía de menor concentración alrededor de la zona de ascenso del agua caliente como sucede en la zona norte del sistema hidrotermal, derivado en parte de la volatilidad del $\mathrm{Hg}_{\text {(ac) }}{ }^{\text {(ac }}$ al estar expuesto a la superficie. Finalmente en los manantiales de menor temperatura (LSA-02, Pozo Araró) queda solo el $\mathrm{Hg}$ remanente.

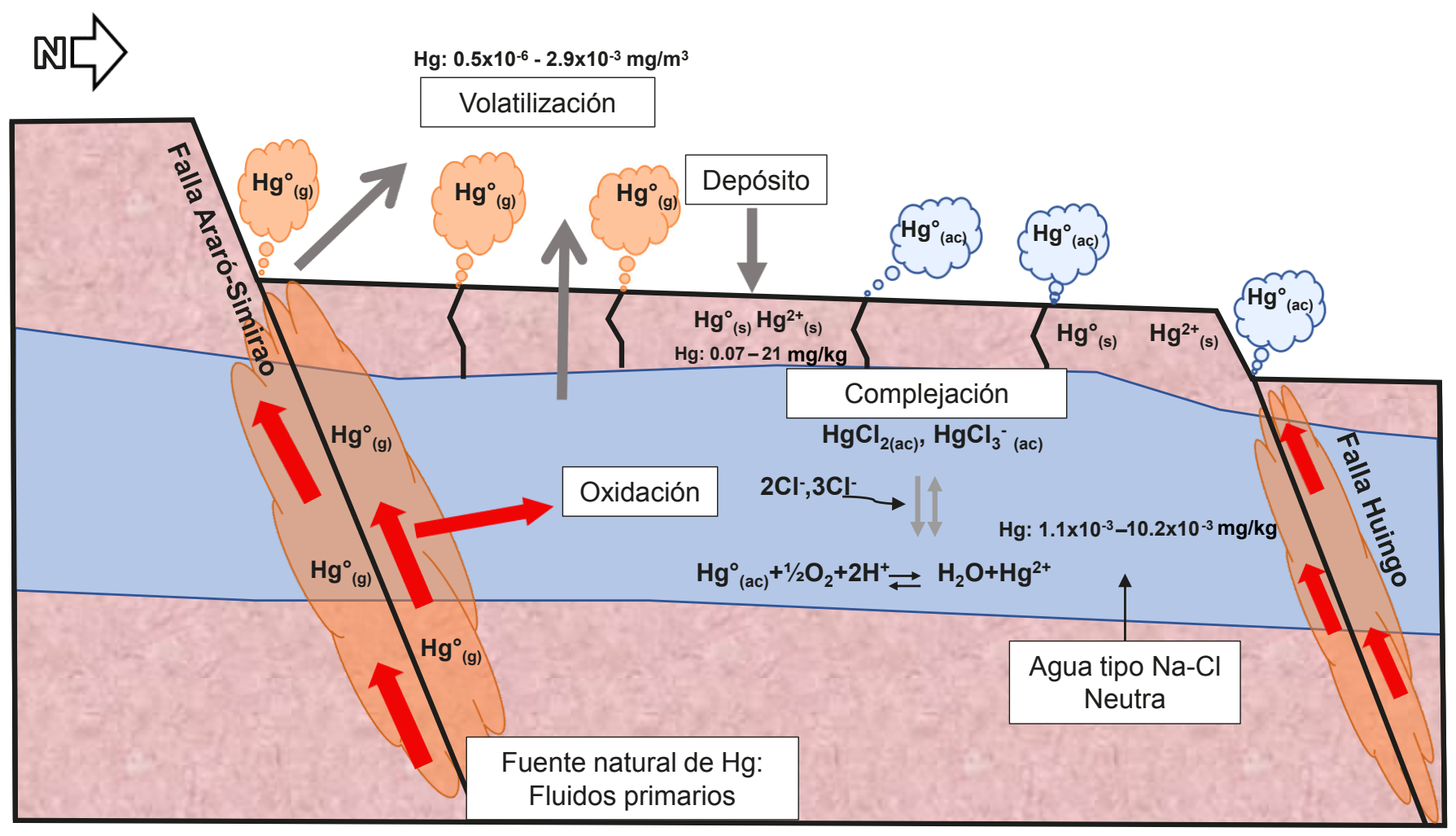

Figura 8. Modelo conceptual del transporte de Hg en el sistema hidrotermal Araró-Simirao. Las fechas rojas indican la dirección de flujo de los fluidos termales y el transporte de $\mathrm{Hg}$, las flechas grises indican los procesos que ocurren en el agua, suelo y atmósfera, y las líneas negras son las trazas de las fallas principales y algunos de los lineamientos en la zona. Las concentraciones de mercurio en el agua y suelo están dadas en $\mathrm{mg} / \mathrm{kg}$. 
Al entrar en contacto los fluidos con el suelo, el depósito de $\mathrm{Hg}$ puede generarse cuando la solución comienza a sobresaturarse en $\mathrm{Hg}^{0}{ }_{(\mathrm{ac})}$ por el decremento en la temperatura (para complejos de bisulfuros), presión (por debajo de la presión de vapor) o $\mathrm{pH}$, y por procesos de oxidación (Barnes et al.,1967); entonces, el Hg es retenido en parte por la formación de quelatos, por su adsorción en arcillas, su afinidad a la materia orgánica o por intercambio iónico (Trost y Bisque, 1971; McNeal y Rose, 1974; Fang, 1978; Landa, 1978) y puede depositarse además, como minerales de sulfuros, hidróxidos o carbonatos de mercurio. En el suelo tiende a estar inmóvil hasta que las condiciones óxido-reductoras se desestabilicen. Derivado de esto, se encontraron altas concentraciones en suelos cercanos a los manantiales fósiles del sistema. Sin embargo, al cambiar las condiciones óxido-reductoras, entre otros parámetros, el mercurio puede ser volatilizado a la atmósfera generando las anomalías de GEM en las zonas de mayor contenido de Hg en suelos.

\section{CONCLUSIONES}

Se encontraron concentraciones anómalas de $\mathrm{Hg}$ en el aire, suelo y agua de manantiales en el sistema geotérmico Araró-Simirao. Las concentraciones anómalas encontradas están asociadas con las zonas de mayor permeabilidad como son las fallas principales. Por ello se sugiere que el $\mathrm{Hg}$ probablemente de origen magmático, se asocia al sistema hidrotermal, que favorece el transporte de mercurio elemental en estado gaseoso hacia la superficie por las zonas de mayor permeabilidad, como es la falla Araró-Simirao, formando aureolas de anomalías. De acuerdo con la distribución de las concentraciones de $\mathrm{Hg}$ en el área, la zona más cercana a los fluidos geotérmicos (ascenso vertical de los gases) es la parte sur del sistema.

El mercurio elemental en estado gaseoso, en su ascenso interacciona con el acuífero subsuperficial, enriqueciendo al acuífero en $\mathrm{Hg}$ y formando complejos clorurados que son transportados en el medio acuoso. Las especies predominantes de $\mathrm{Hg}$ en agua son $\mathrm{Hg}^{0}{ }_{\text {(ac) }}, \mathrm{HgCl}_{2}$ y $\mathrm{HgCl}_{3}$, aunque la abundancia de $\mathrm{Hg}^{0}{ }_{(\mathrm{ac})}$ es menor con respecto a $\mathrm{Hg}^{2+}$. Al descargar a la superficie el agua termal enriquecida en $\mathrm{Hg}$, éste se volatiliza como $\mathrm{Hg}_{(\mathrm{g})}^{0}$ y permanece en la atmósfera o puede depositarse en los suelos aledaños a los manantiales adhiriéndose a partículas de materia orgánica.

De forma general, se puede inferir que los procesos geoquímicos que se llevan a cabo para el transporte de $\mathrm{Hg}$ entre los compartimentos ambientales: agua, suelo y atmósfera, en el sistema hidrotermal son: volatilización, oxidación, complejación y depósito. Sin embargo, es necesario identificar con precisión las especies químicas (compuestos organometálicos del mercurio y complejos clorurados) descritas en el presente trabajo empleando técnicas analíticas más especializada (p. ej., electroquímica analítica) para corroborar el modelo conceptual del transporte de $\mathrm{Hg}$ en la zona de estudio.

\section{AGRADECIMIENTOS}

Los autores expresan su agradecimiento a la Ing. Donaji García, Ing. Jaziel Froylan y QFB Sergio Cruz por su apoyo en el trabajo de campo, a la QBP Blanca Xóchitl Felipe por su apoyo en los análisis químicos de agua. Este trabajo fue financiado por el proyecto P02 del CeMIE-GEO del CONACYT-SENER-Fondo de Sustentabilidad Energética \#207032-2013-04 y el proyecto interno N-123 del Instituto de Geofísica de la Universidad Nacional Autónoma de México.

\section{REFERENCIAS}

Adriano, D.C., 2001, Trace elements in terrestrial environments: New York, Springer-Verlag. 867 pp.

APHA, 1998, Standard Methods for the Examination of Water and Wastewater: Washington DC, American Public Health Association, 874 pp.

Ariya, P.A., Skov, H., Grage, M.M.-L., Goodsite M.E., 2008, Gaseous Elemental Mercury in the Ambient Atmosphere: Review of the Application of Theoretical Calculations and Experimental Studies for Determination of Reaction Coefficients and Mechanisms with Halogens and Other Reactants: Advances in Quantum Chemistry, 55, 43-55.

Ávalos-Tapia, D., 2016, Reconocimiento de las asociaciones minerales de alteración y depósitos hidrotermales relacionados con la actividad de los manantiales termales de Araró, Michoacán: Universidad Autónoma de Nuevo León, Facultad de Ciencias de la Tierra, Tesis de licenciatura, 89 pp.

Bagnato, E., Viveiros, F., Pacheco, J.E., D’Agostino, F.D., Silva, C., Zanon, A., 2018, $\mathrm{Hg}$ and $\mathrm{CO}_{2}$ emissions from diffuse degassing and fumaroles at Furnas Volcano (Sao Miguel Island, Azores): Gas flux and thermal energy output: Journal of Geochemical Exploration, 190, 39-57.

Bagnato, E., Aiuppa, A., Parello F., Calabrese S., D'Alessandro W., Mather T.A., McGonigle A.J.S., Pyle D.M., Wänberg I., 2007, Degassing of gaseous (elemental and reactive) and particulate mercury from Mount Etna volcano (Southern Italy): Atmospheric Environment, 41, 7377-7388, doi: 10.1016/j.atmosenv.2007.05.060

Barnes, H.L., Romberger, S.B., Stemprok, M, 1967, Ore solution chemistry II. Solubility of HgS in sulfide solutions: Economic Geology, 62, 957-982.

Beckers F., Rinklebe J., 2017, Cycling of mercury in the environment: Sources, fate, and human health implications: A review, Critical Reviews in Environmental Science and Technology, 47(9), 693-794, DOI: 10.1080/10643389.2017.1326277

Cabassi, J., Tassi, F., Venturi, S., Calabrese, S., Capecchiacci, F., DÁlessandro, W., Vaselli, O., 2017, A new approach for the measurement of gaseous elemental mercury (GEM) and $\mathrm{H}_{2} \mathrm{~S}$ in air from anthropogenic and natural sources: Examples from Mt. Amiata (Siena, Italy) and Solfatara Crater (Campi Flegrei, Southern Italy): Journal of Geochemical Exploration, $175,48-58$.

Canet, C., Rodríguez-Díaz, A.A., Bernal, I.D., Pi, T., Sánchez Córdova, M.M., Núñez Useche, F., Villanueva Estrada, R., Molina, G., Reich, M., Peláez, B., Jiménez Salgado, E., González Partida, E., Sandoval Medina, F., Carrillo Sánchez, C.B., 2019, Consideraciones sobre el sistema geotérmico de San Bartolomé de los Baños, Guanajuato (México), desde un análisis de la alteración hidrotermal y las inclusiones fluidas: Geofísica Internacional, 58(3), 229-246.

Canet, C., Prol-Ledesma, R.M., Proenza, J., Rubio-Ramos, M. A., Forrest, M., Torres-Vera, M.A., Rodríguez-Díaz, A.A., 2005, Mn-Ba-Hg Mineralization at shallow submarine hydrothermal vents in Bahía Concepción, Baja California Sur, Mexico: Chemical Geology, 224, 96-112.

Cram, S., Galicia, L., Israde-Alcántara I., 2010, Atlas de la cuenca del lago de Cuitzeo. Análisis de su geografía y entorno socioambiental: México, Universidad Nacional Autónoma de México, Universidad Michoacana de San Nicolás de Hidalgo, 314 pp.

Custodio, E., Llamas, M.R., 1996, Hidrología subterránea: Barcelona, Ediciones Omega S.A., 2 tomos, $2^{\text {a }}$ ed., 2418 pp.

D'Amore, F., Scandiffio, G., Panichi, C., 1983, Some observations on the chemical classification of ground waters: Geothermics, 12, 141-148, doi: 10.1016/0375-6505 (83)90024-X

DETENAL (Dirección General de Estudios del Territorio Nacional), 1978, Carta geológica Zinapécuaro E14A14: México, 1 mapa.

Engle, M.A., Gustin M.S., Goff, F., Counce D.A., Janik C.J., Bergfeld, D., Rytuba J.J., 2006, Atmospheric mercury emissions from substrates and fumaroles associated with three hydrothermal systems in the western United States: Journal of Geophysical Research, 111, D17304, doi:10.1029/2005JD006563, 2006

Fang, 1978, Sorption and transformation of mercury vapor by dry soil: Environmental Science Technology, 12(3), 285-288.

Fein J.B., Williams-Jones A.E., 1997, The role of mercury-organic interactions in the hydrothermal transport of mercury: Economic Geology, 92(1), 20-28.

Garduño-Monroy, V.H., Gutiérrez-Negrín, L.C.A., 1992, Magmatismo, hiatus y tectonismo de la Sierra Madre Occidental y del Cinturón Volcánico Mexicano: Geofísica Internacional, 31(4), 417-429.

Gustin, M.S., 2003, Are mercury emission from geologic sources significant? A status report: Science of Total Environment, 304(1-3), 153-167.

Gustin, M.S., Lindberg, S., Marsik, F., Casimir, A., Ebinghaus, R., Edwards, G., Hubble-Fitzgeral, C., Kemp, H.H., Leonard, T., London, J., Majewski, M., Montecinos, M., Owens, J., Pilote, M., Poissant, L., Rasmussen, P., Schaedlich, F., Schneeberger, D., Schroedet, W., Sommar, J., Turner, R., Vette, A., Wallschlaeger, D., Xiao, Z., Zhang, H., 1999, Nevada STORMS project: Measurement of Mercury emissions from naturally enriched 
surfaces: Journal of Geophysical Research, 104 (D17), 21831-21844.

Gustin, M.S., Taylor, G.E., Maxey, R.A., 1997, Effect of temperature and air movement on the flux elemental mercury from substrate to the atmosphere: Journal of Geophysical Research, 102 (D3), 3891-3898.

Han, F.X., 2007, Section II: Biogeochemistry of trace elements in arid environments, en Alloway, B.J., Trevors, J.T., (eds.) Biogeochemistry of Trace Elements in Arid Environments; Dordrecht, Springer-Verlag, 368 pp.

Hazen, R., Golden, J., Downs, R., Hystad, G., Grew, E., Azzolini, D., Sverjensky, D., 2012, Mercury (Hg) mineral evolution: A mineralogical record of supercontinent assembly, changing ocean geochemistry, and the emerging terrestrial biosphere: American Mineralogist, 97, 1013-1042, DOI: 10.2138/am.2012.3922

Hem, J.D., 1970, Chemical behavior of mercury in aqueous media. In: Mercury in the environment: U.S. Geological Survey Professional Paper 713, 9, 19-24.

Henneberger, R.C., 1986, Ohakuri fossil epithermal system, en Henley, R.W., Hedenquist, J.W., Roberts P.J. (eds.), Guide to the Active Epithermal (Geothermal) Systems and Precious Metal Deposits of New Zealand: Berlin, Monograph Series on Mineral Deposits, Gebrüder Borntraeger, 26, 121-128.

Israde-Alcántara, I., Velázquez-Durán, R., Lozano, S. Bischoff, J., Domínguez, G., Garduño, V., 2010, Evolución paleolimnológica del Lago de Cuitzeo, Michoacán durante el Pleistoceno-Holoceno: Boletín de la Sociedad Geológica Mexicana, 62(3), 345-357.

Israde-Alcántara I., Garduño-Monroy V.H., 1999, Lacustrine record in a volcanic intra-arc setting: The evolution of the Late Neogene Cuitzeo basin system (central-western Mexico, Michoacan): Palaeogeography, Palaeoclimatology, Palaeoecology Journal, doi: 10.1016/S0031-0182(99)00024-3

Jiménez-Moreno, M., Perrot, V., Epov, V.N., Monperrus, M., Amoroux, D., 2013, Chemical Kinetic isotope fractionation of mercury during abiotic methylation of $\mathrm{Hg}(\mathrm{II})$ by methylcobalamin in aqueous media: Chemical Geology, 336, 26-36, https://doi.org/10.1016/j.chemgeo.2012.08.029

Kabata-Pendias, A, 2011, Trace elements in soils and plants: Boca Raton, FL, CRC Press, 520 pp.

Landa, E.R., 1978, The retention of metallic mercury vapor by soils: Geochimica et Cosmochimica Acta, 42(9), 1407-1411.

Leal-Acosta, M.L., Shumilin, E., Mirlean, N., Sapozhnikov D., Gordeev, V., 2010, Arsenic and mercury contamination of sediments of geothermal springs, mangrove lagoon and the Santispac bight, Bahía Concepción, Baja California Peninsula: Bulletin of Environmental Contamination and Toxicology 85, 609-613, https://doi.org/10.1007/s00128-010-0135-5

Leal-Acosta, M.L., Shumilin, E., Mirlean, N., Delgadillo-Hinojosa, F., SánchezRodríguez, I., 2013, The impact of marine shallow-water hydrothermal venting on arsenic and mercury accumulation by seaweed Sargassum sinicola in Concepcion Bay, Gulf of California: Environmental Science: Processes and Impact, 15(2), 470-477, DOI: 10.1039/c2em30866e

Leopold, K., Foulkes, M., Worsfold, P., 2010, Methods for the determination and speciation of mercury in natural waters - a review: Analytica Chimica Acta, 663, 127-138, doi: 10.1016/j.aca.2010.01.048

Lindberg, S., Bullock, R., Ebinghaus, R., Engstrom, D., Feng, X., Fitzgerald, W., Pirrone, N., Prestbo, E., Seignur, C., 2007, A synthesis of Progress and Unertainties in attributing the sources of Mercury in Deposition: Ambio, 36(1), 19-32.

Lindqvist, O., Rodhe, H., q1985, Atmospheric mercury- a review: Tellus B, 37B(3), 136-159, https://doi.org/10.1111/j.1600-0889.1985.tb00062.x

Lindqvist O., Johansson K., Bringmark L., Timm B., Aastrup M., Andersson A., Hovsenius G., Håkanson L., Iverfeldt A., Meili M., 1991, Mercury in the Swedish environment. Recent research on causes, consequences and corrective methods: Water, Air, and Soil Pollution, 55, xi-261, https://doi. org/10.1007/BF00542429

McNeal, J.M., Rose, A.W., 1974, The geochemistry of mercury in sedimentary rocks and soils in Pennsylvania: Geochimica et Cosmochimica Acta, 38(12), 1759-1784.

Nicholson, K., 1993, Geothermal Fluids; Chemistry and Exploration Techniques: Berlin, Springer Verlag, 263 pp., doi: 10.1007/978-3-642-77844-5

NOM-127-SSA1-1994, Salud ambiental, agua para uso y consumo humanolímites permisibles de calidad y tratamientos a que debe someterse el agua para su potabilización. Norma Oficial Mexicana disponible en: http://www. salud.gob.mx/unidades/cdi/nom/127ssa14.html
Olvera-García, E., Garduño-Monroy, V.H., Liotta, D., Brogi, A., BermejoSantoyo, G., Guevara-Alday, J.A., 2020, Neogene-Quaternary normal and transfer faults controlling Deep-seated geothermal systems: The case of San Agustín del Maíz (central Trans-Mexican Volcanic Belt, México): Geothermics, 86, 101791.

Pastrana-Corral, M.A., Wakida, F.T., García-Flores, E., Rodríguez-Mendevil D.D., Quiñonez-Plaza A., Piñon-Colín T.D.J., 2016, Soil mercury levels in the area surrounding the Cerro Prieto geothermal complex, MEXICO: Environmental Monitoring and Assessment, 188, 466 (2016), https://doi. org/10.1007/s10661-016-5474-1

Phuong N.K., Harijoko A., Itoi R., Unoki Y., 2012, Water geochemistry and soil gas survey at Ungaran geothermal field, central Java, Indonesia: Journal of Volcanology and Geothermal Research, 229-230, 23-33, doi: 10.1016/j. jvolgeores.2012.04.004

Pérez-Martínez, I., Villanueva-Estrada, R.E., Rodríguez-Díaz, A.A., Canet, C., García, R., Ramos-Leal, J.A., García, D., Cambrón, J.F., 2021, Diffuse gases in soil of Araró-Simirao geothermal system, Michoacán, Mexico: Andean Geology, 48(3), DOI:10.5027/andgeoV48n3-3327

Pola, A., Martínez-Martínez, J., Macías, J.L., Fusi, N., Crosta, G., GarduñoMonroy, V.H., Núñez-Hurtado, J.A., 2016, Geomechanical characterization of the Miocene Cuitzeo ignimbrites, Michoacán, Central Mexico: Engineering Geology, 214, 79-93.

Prol-Ledesma, R.M., Morán-Zenteno, D.J. 2019, Heat flow and geothermal provinces in Mexico: Geothermics, 78, 183-200.

Prol-Ledesma, R.M., Canet, C., Melgarejo, J., Tolson, G., Rubio Ramos, M., Cruz-Ocampo, J.C., Ortega-Osorio, A., Torres-Vera, M.A., Reyes, A., 2002, Cinnabar deposition in submarine coastal hydrothermal vents, Pacific margin of central Mexico: Economic Geology, 97, 1331-1340, DOI:10.2113/97.6.1331

Prol-Ledesma, R.M., Carrillo-de la Cruz, J.L., Torres-Vera, M. A., MembrilloAbad, A.S., Espinoza-Ojeda, O.M., 2018, Heat flow map and geothermal resources in Mexico, Mapa de flujo de calor y recursos geotérmicos de México: Terra Digitalis, 2(2), 1-38.

Rasmussen, P.E., Edwards, G.C., Kemp, J.R., Fitzgerald-Hubble C.R., Schroeder, W.H., 1998, Towards an improved natural sources inventory for mercury, en Skeaff, J. (ed.), Metals and the Environment: An International Symposium, Montreal: Canadian Institute of Mining, Metallurgy and Petroleum , 73-83.

Schroeder W.H., Munthe J., 1998, Atmospheric mercury-An overview: Atmospheric environment, 32(5), 809-822.

Segovia, N., Barragán, R.M., Tello, E., Alfaro, R., Mena, M., 2005a, Geochemical Characteristics and 222Rn Measurements at Cuitzeo Basin (Mexico) Thermal Springs and Artesian Wells, en Proceedings World Geothermal Congress 2005, Antalya, Turkey, 6 pp.

Segovia, N., Barragán, R.M., Tello, E., Alfaro, R., Mena, M., Pulinets, S., Leyva, A., 2005b, Geochemical exploration at Cuitzeo Basin Geothermal Zone (Mexico): Journal of Applied Sciences, 5(9), 1658-1664.

Segovia, N., Barragán, R.M., Alfaro, R., Arellano V.M., Mena, M. 2010, Fluid-mineral equilibrium of spring Waters at Cuitzeo Basin (Mexico) geothermal zone, en Proceedings World Geothermal Congress 2010, Bali, Indonesia, $7 \mathrm{pp}$.

Slemr, F., Scheel, H.E., 1985, Distribution, speciation, and budget of atmospheric mercury: Journal of Atmospheric Chemistry, 3(4), 407-434, DOI: 10.1007/ BF00053870

Selin, N., 2009, Global Biogeochemical Cycling of Mercury: A Review: Annual Review of Environment and Resources 34, 43-63, doi: 10.1146/annurev. environ.051308.084314

SMN (Servicio Meteorológico Nacional) 2019, Climogramas, (en línea) disponible en: https://smn.conagua.gob.mx/es/climatologia/informacionclimatologica/climogramas-1981-2010, consultado el 11 de septiembre de 2019.

Steenhuisen, F., Wilson, S.J., 2019, Development and application of an updated geospatial distribution model for gridding 2015 global mercury emissions: Atmospheric environment, 211, 138-150, https://doi.org/10.1016/j. atmosenv.2019.05.003

Sun, Y., Guo, Z., Du, J., Zhao, W., 2020, Diffuse emission and transport of gaseous elemental mercury (GEM) in the Mapamyum geothermal system, Western Tibet (China): Journal of Volcanology and Geothermal Research, 397, 106825.

Trost P.B., Bisque R.E., 1971, Differentiation of vaporous and ionic $\mathrm{Hg}$ in soils, 
en 3rd International Geochemical. Exploration. Symposium, Proceedings, Toronto, Canadá: Canadian Institute of Mining and Metallurgy, 11, 276-278.

USEPA (United States Environmental Protection Agency) 1997, Mercury Study Report to Congress, Volume III. Fate and Transport of Mercury in the Environment: Office of Air Quality Planning \& Standards and Office of Research and Development, EPA-452/R-97-005.

USGS (United States Geological Survey), 1970, Mercury in the Environment: Washington, U.S., Geological Survey Professional Paper 713, 67 pp.

Varekamp, J.C., Buseck, P.R., 1986, Global mercury flux volcanic and geothermal sources: Applied Geochemistry, 1, 65-73.

Varekamp, J.C., Buseck, P.R., 1984, The speciation of mercury in hydrothermal systems, with applications to ore deposition: Geochimica et Cosmochimica Acta, $48,177-185$.

Varekamp, J.C., Buseck, P.R., 1983, Hg anomalies in soils: A geochemical exploration method for geothermal areas: Geothermics, 12(1), 29-47.
Viggiano Guerra J.C., Gutiérrez Negrín, L.C.A., 2005, The geothermal system of Araró, Mexico, as an independent system of Los Azufres, en Proceedings World Geothermal Congress, Antalya, Turkey, 7 pp.

Viggiano Guerra J.C., Gutiérrez Negrín L.C.A., 2003, Régimen de flujo hidrotermal en la zona geotérmica de Araró, Michoacán, México: Ingeniería Hidráulica en México, XVII(1), 39-53.

Yu, Q., Luo, Y., Wang, S., Wang, Z., Hao, J. Dan, L., 2018, Gaseous elemental mercury (GEM) fluxes over canopy of two typical subtropical forest in south China: Atmospheric Chemistry and Physics, 18. 495-509, https:// doi.org/10.5194/acp-18-495-2018

Manuscrito recibido: agosto 7, 2020

Manuscrito corregido recibido: abril 30, 2021

Manuscrito aceptado: mayo 24, 2021 